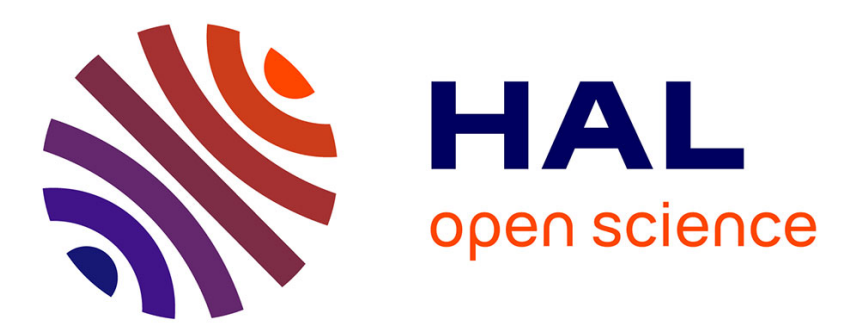

\title{
Qualitative identification of cracks using 3D transient elastodynamic topological derivative: formulation and FE implementation
}

\author{
Cédric Bellis, Marc Bonnet
}

\section{- To cite this version:}

Cédric Bellis, Marc Bonnet. Qualitative identification of cracks using 3D transient elastodynamic topological derivative: formulation and FE implementation. Computer Methods in Applied Mechanics and Engineering, 2013, 253, pp.89-105. 10.1016/j.cma.2012.10.006 . hal-00741515

\author{
HAL Id: hal-00741515 \\ https://hal.science/hal-00741515
}

Submitted on 13 Oct 2012

HAL is a multi-disciplinary open access archive for the deposit and dissemination of scientific research documents, whether they are published or not. The documents may come from teaching and research institutions in France or abroad, or from public or private research centers.
L'archive ouverte pluridisciplinaire HAL, est destinée au dépôt et à la diffusion de documents scientifiques de niveau recherche, publiés ou non, émanant des établissements d'enseignement et de recherche français ou étrangers, des laboratoires publics ou privés. 


\title{
Qualitative identification of cracks using 3D transient elastodynamic topological derivative: formulation and FE implementation
}

\author{
Cédric BELLIS ${ }^{(\mathrm{a}) *}{ }^{*}$ Marc BONNET ${ }^{(\mathrm{b}) \dagger}$ \\ (a) Solid Mechanics Laboratory (UMR CNRS 7649), École Polytechnique, Palaiseau, France \\ (b) POems (UMR 7231 CNRS-ENSTA-INRIA), ENSTA, Paris, France
}

\begin{abstract}
A time-domain topological derivative (TD) approach is developed for transient elasticwave imaging of buried cracks. The TD, which quantifies the sensitivity of the misfit cost functional to the creation at a specified location of an infinitesimal trial crack, is expressed in terms of the time convolution of the free field and an adjoint field as a function of that specified location and of the trial crack shape. Following previous studies on cavity identification in similar conditions, the TD field is here considered as a natural and computationally efficient approach for defining a crack location indicator function. This study emphasizes the implementation and exploitation of TD fields using the standard displacement-based FEM, a straightforward exploitation of the relevant sensitivity formulation established here. Results on several numerical experiments on 3D elastodynamic and acoustic configurations are reported and discussed, allowing to assess and highlight many features of the proposed TD-based fast qualitative crack identification, including its ability to identify multiple cracks and its robustness against data noise.
\end{abstract}

Key words : Crack, inverse scattering, topological derivative, adjoint field method

\section{INTRODUCTION}

The reconstruction of cracks buried in solids using transient elastic waves is a classical inverse problem that arises in a number of applications such as nondestructive material testing or seismic imaging $[20,52]$. Crack identification requires data on the scattering of waves by the sought $\operatorname{crack}(\mathrm{s})$, obtained from measurements, that is overdetermined compared to the conditions under which the forward elastodynamic problem for an assumed crack configuration is well-posed [17]. Over the past two decades, the theory of inverse scattering have experienced a paradigm shift through the development of qualitative, non-iterative methods $[23,39,47]$ that consist in formulating and computing indicator functions for the hidden flaw location and geometry. This type of approach is of particular relevance in 3D configurations where minimization-based algorithms entail high computational costs due to repeated use of elastodynamic forward solvers.

Non-iterative approaches relevant to elastodynamic crack identification notably include methods based on the concepts of linear sampling [22,27,46], reciprocity gap [12,14, 21], or topological derivative, the latter being considered herein. Initially introduced in connection with structural topology optimization [31,51], the concept of topological derivative (TD) has, since then, also been applied as a convenient, computationally economical means for qualitative flaw identification, see e.g. [40] for 2D potential problems or [19] for 3D frequency-domain

\footnotetext{
${ }^{*}$ Current address: Dept. of Appl. Phys. and Appl. Math., Columbia University, New York NY 10027, USA

${ }^{\dagger}$ Corresponding author. E-mail: mbonnet@ensta.fr
} 
elastodynamics. Strong connections exist with asymptotic methods [4] that establish and exploit small-defect asymptotic expansions of solutions, and more generally with the asymptotic treatment of geometrical singular perturbations [43, 44].

The topological derivative analysis has been extended to identification problems under transient dynamical conditions $[5,11,16]$ and to the identification of various kinds of inhomogeneities [6,33,36]. Moreover, analogies between TD and time reversal have been investigated $[29,54]$. On the other hand, only scattered studies have so far been devoted to crack identification, including [10] for 2D potential problems, [7] and [9] for the 2D and 3D Helmholtz equation, respectively, and [32] in 2D elastostatics. Following up on recent work [13] addressing cavity identification using a 3D transient elastodynamic topological derivative formulation, this article proposes a non-iterative, qualitative approach to crack identification in the same context. The topological derivative with respect to the nucleation of small trial cracks is established for a broad class of cost functionals, which includes the usual least-squares misfit functionals often used for identification. Both the elastodynamic and the acoustic cases are treated, with emphasis on the former. An adjoint-based approach provides a simple and efficient formulation, which is implemented within a standard FEM environment and demonstrated on several sample 3D transient problems. To the authors' best knowledge, this study is the first attempt at applying the topological derivative to 3D crack identification from transient data.

The article is organized as follows. The derivation of the topological derivative, based on a small-crack asymptotic analysis, is presented in Sections 2 (elastodynamics) and 3 (acoustics), with the relevant elastic moment tensors given in closed form for circular or elliptical trial crack shapes and some technical steps deferred to appendices. The proposed TD-based qualitative identification approach and its implementation are described in Section 4. Then, results on numerical experiments are reported and discussed in Section 5.

Crack identification problem. A linearly elastic solid body, containing a crack (or a set thereof) to be identified, is interrogated by means of a dynamic excitation. The solid material is characterized by its mass density $\rho$ and elasticity tensor $\mathcal{C}$ which, for an isotropic material, is given in terms of the shear modulus $\mu$ and Poisson's ratio $\nu$ by

$$
\mathcal{C}=2 \mu\left(\frac{\nu}{1-2 \nu} \boldsymbol{I} \otimes \boldsymbol{I}+\boldsymbol{I}_{\mathrm{sym}}\right)
$$

$\boldsymbol{I}$ being the second-order identity tensor and $\boldsymbol{I}_{\text {sym }}$ the symmetric fourth-order identity tensor. Let $\Omega \subset \mathbb{R}^{3}$ and $\Gamma$ denote the open domain occupied by the reference (uncracked) solid and its boundary, respectively. To identify hidden cracks, transient excitations are imposed in the form of tractions applied over $\Gamma_{\mathrm{N}} \subset \Gamma$ and displacements prescribed on the complementary external surface $\Gamma_{\mathrm{D}}=\Gamma \backslash \Gamma_{\mathrm{N}}$, with initial rest conditions assumed at time $t=0$. Letting $S$ denote a trial crack, the prescribed excitation gives rise to elastodynamic displacement fields $\boldsymbol{u}$ (the free field) in the reference domain $\Omega$ and $\boldsymbol{u}_{S}$ in the cracked domain $\Omega_{S}=\Omega \backslash \bar{S}$.

The identification of the actual crack (or set of cracks) $S^{\star}$ is here based on exploiting overdetermined data on the external boundary. More specifically, the measured value $\boldsymbol{u}_{\text {obs }}$ of the displacement $\boldsymbol{u}^{\star}:=\boldsymbol{u}_{S^{\star}}$ induced in the actual flawed solid $\Omega^{\star}:=\Omega_{S^{\star}}$ by the prescribed excitation is assumed to be available on the observation surface $\Gamma_{\text {obs }} \subset \Gamma_{\mathrm{N}}$ and during the time interval $[0, T]$ (other possibilities can be considered with minimal changes to the formulation). The discrepancy between a trial configuration $\Omega_{S}$ and the correct configuration $\Omega^{\star}$ is evaluated by means of a cost functional $\mathbb{J}$ defined in terms of a misfist density function $\varphi$ :

$$
\mathbb{J}\left(\Omega_{S}, T\right)=\int_{0}^{T} \int_{\Gamma_{\mathrm{obs}}} \varphi\left(\boldsymbol{u}_{S}(\boldsymbol{\xi}, t), \boldsymbol{\xi}, t\right) \mathrm{d} S_{\xi} \mathrm{d} t .
$$


The misfit density $\varphi$ is chosen so as to define a measure of the gap between measurements $\boldsymbol{u}_{\text {obs }}$ and the displacement $\boldsymbol{u}_{S}$ arising in a trial cracked solid $\Omega_{S}$. Numerical experiments presented in this work are based on the commonly-used least squares misfit function:

$$
\varphi(\boldsymbol{w}, \boldsymbol{\xi}, t)=\frac{1}{2}\left\|\boldsymbol{w}-\boldsymbol{u}^{\mathrm{obs}}(\boldsymbol{\xi}, t)\right\|^{2} .
$$

In that case, the cost functional (2) is equivalent (by Parseval's theorem) to a sum over frequencies of least-squares cost functionals on displacements solving time-harmonic problems, see e.g. $[29,49]$.

\section{ELASTIC TOPOLOGICAL DERIVATIVE}

\subsection{Preliminaries}

The topological derivative of the cost functional (2) is here defined with reference to the creation of an infinitesimal crack of characteristic size $a$ at a given location $\boldsymbol{z} \in \Omega$, defined by $S_{a, \boldsymbol{z}}(\boldsymbol{z}):=$ $\boldsymbol{z}+a \mathcal{S}$ in terms of a fixed normalized open surface $\mathcal{S}$ containing the origin and specifying a shape (e.g. $\mathcal{S}$ is a unit disk for a nucleating penny-shaped crack) and orientation for the crack. The corresponding trial cracked solid $\Omega_{a, z}$ is thus defined by $\Omega_{a, z}:=\Omega \backslash \bar{S}_{a, \boldsymbol{z}}$. Following earlier works on topological derivative, e.g. $[19,33,51]$, one seeks the asymptotic behavior of $\mathbb{J}\left(\Omega_{a, z}, T\right)$ as $a \rightarrow 0$ through the expansion:

$$
\mathbb{J}\left(\Omega_{a, \boldsymbol{z}}, T\right)=\mathbb{J}(\Omega, T)+\eta(a) \mathbb{T}(\boldsymbol{z} ; \mathcal{S}, T)+o(\eta(a)) \quad(a \rightarrow 0)
$$

where the function $\eta(a)$, to be determined, vanishes in the limit $a \rightarrow 0$ and the topological derivative $\mathbb{T}(\boldsymbol{z} ; \mathcal{S}, T)$ is a function of the sampling point $\boldsymbol{z}$, trial crack shape $\mathcal{S}$, and experiment duration $T$.

The prescribed dynamical loading applied on $\Omega_{a, \boldsymbol{z}}$ gives rise to an elastodynamic state $\boldsymbol{u}_{a, \boldsymbol{z}}$ that can be conveniently decomposed into $\boldsymbol{u}_{a, \boldsymbol{z}}=\boldsymbol{u}+\boldsymbol{v}_{a, \boldsymbol{z}}$, where the free-field $\boldsymbol{u}$ is the response of the reference domain $\Omega$ while the scattered field $\boldsymbol{v}_{a, z}$ is governed by the initial-boundary value problem (IBVP)

$$
\begin{array}{ll}
\mathcal{L} \boldsymbol{v}_{a, \boldsymbol{z}}(\boldsymbol{\xi}, t)=\mathbf{0} & \left(\boldsymbol{\xi} \in \Omega_{a, \boldsymbol{z}}, t \geq 0\right) \\
\boldsymbol{t}^{ \pm}\left[\boldsymbol{v}_{a, \boldsymbol{z}}^{ \pm}\right](\boldsymbol{\xi}, t)=-\boldsymbol{t}^{ \pm}[\boldsymbol{u}](\boldsymbol{\xi}, t) & \left(\boldsymbol{\xi} \in S_{a, \boldsymbol{z}}^{ \pm}, t \geq 0\right) \\
\boldsymbol{t}\left[\boldsymbol{v}_{a, \boldsymbol{z}}\right](\boldsymbol{\xi}, t)=\mathbf{0} & \left(\boldsymbol{\xi} \in \Gamma_{\mathrm{N}}, t \geq 0\right) \\
\boldsymbol{v}_{a, \boldsymbol{z}}(\boldsymbol{\xi}, t)=\mathbf{0} & \left(\boldsymbol{\xi} \in \Gamma_{\mathrm{D}}, t \geq 0\right) \\
\boldsymbol{v}_{a, \boldsymbol{z}}(\boldsymbol{\xi}, 0)=\dot{\boldsymbol{v}}_{a, \boldsymbol{z}}(\boldsymbol{\xi}, 0)=\mathbf{0} & \left(\boldsymbol{\xi} \in \Omega_{a, \boldsymbol{z}}\right),
\end{array}
$$

where $\boldsymbol{\xi}$ and $t$ denotes the position vector and time, $\mathcal{L}$ is the Navier space-time partial differential operator defined by $\mathcal{L} \boldsymbol{w}:=\operatorname{div}(\boldsymbol{\sigma}[\boldsymbol{w}])-\rho \ddot{\boldsymbol{w}}\left(\right.$ with $\boldsymbol{\sigma}[\boldsymbol{w}]=\mathcal{C}: \boldsymbol{\nabla} \boldsymbol{w}=\mathcal{C}:\left(\boldsymbol{\nabla} \boldsymbol{w}+(\boldsymbol{\nabla} \boldsymbol{w})^{\mathrm{T}}\right) / 2$ denoting the elastic stress tensor associated with a displacement $\boldsymbol{w}), \boldsymbol{v}_{a, \boldsymbol{z}}^{ \pm}$are the scattered displacements on the crack faces $S_{a, \boldsymbol{z}}^{ \pm}$, and the operator $\boldsymbol{w} \mapsto \boldsymbol{t}[\boldsymbol{w}]=\boldsymbol{\sigma}[\boldsymbol{w}] \cdot \boldsymbol{n}\left(\operatorname{resp} . \boldsymbol{w} \mapsto \boldsymbol{t}^{ \pm}[\boldsymbol{w}]=\boldsymbol{\sigma}[\boldsymbol{w}] \cdot \boldsymbol{n}^{ \pm}\right)$ yields the traction vector associated with a displacement $\boldsymbol{w}$ and the outward normal $\boldsymbol{n}$ on $\Gamma$ (resp. $\boldsymbol{n}^{ \pm}$on $S_{a, \boldsymbol{z}}^{ \pm}$). In (5) and hereinafter, the symbols "." and ":" denote single and double dot products, with the convention $\boldsymbol{a}: \boldsymbol{b}=a_{i j} b_{i j}$ used in the latter case.

Expanding the cost functional (2) about $\boldsymbol{u}_{a, \boldsymbol{z}}=\boldsymbol{u}$ to first order w.r.t. $\boldsymbol{v}_{a, \boldsymbol{z}}=\boldsymbol{u}_{a, \boldsymbol{z}}-\boldsymbol{u}$, one obtains

$$
\begin{aligned}
& \mathbb{J}\left(\Omega_{a, \boldsymbol{z}}, T\right)=\mathbb{J}(\Omega, T)+\int_{0}^{T} \int_{\Gamma_{\mathrm{obs}}} \frac{\partial \varphi}{\partial \boldsymbol{u}}[\boldsymbol{u}(\boldsymbol{\xi}, t), \boldsymbol{\xi}, t] \cdot \boldsymbol{v}_{a, \boldsymbol{z}}(\boldsymbol{\xi}, t) \mathrm{d} S_{\xi} \mathrm{d} t+o\left(\left\|\boldsymbol{v}_{a, \boldsymbol{z}}\right\|_{L^{2}\left(\Gamma_{\mathrm{obs}} \times[0, T]\right)}\right) \\
& (a \rightarrow 0)
\end{aligned}
$$


Then, since the scattered field is expected to vanish for infinitesimal cracks, i.e. $\lim _{a \rightarrow 0}\left\|\boldsymbol{v}_{a, \boldsymbol{z}}\right\|=0$, the topological derivative $\mathbb{T}(\boldsymbol{z} ; \mathcal{S}, T)$ and the leading asymptotic behavior $\eta(a)=o\left(\left\|\boldsymbol{v}_{a, \boldsymbol{z}}\right\|_{L^{2}\left(\Gamma_{\mathrm{obs}} \times[0, T]\right)}\right)$ featured in (4) are to be found by identification from

$$
\int_{0}^{T} \int_{\Gamma_{\mathrm{obs}}} \frac{\partial \varphi}{\partial \boldsymbol{u}}[\boldsymbol{u}(\boldsymbol{\xi}, t), \boldsymbol{\xi}, t] \cdot \boldsymbol{v}_{a, \boldsymbol{z}}(\boldsymbol{\xi}, t) \mathrm{d} S_{\xi} \mathrm{d} t=\eta(a) \mathbb{T}(\boldsymbol{z} ; \mathcal{S}, T)+o(\eta(a)) \quad(a \rightarrow 0) .
$$

This requires finding the leading asymptotic behavior as $a \rightarrow 0$ of the left-hand side of (6). One possible way, along the lines of the so-called direct differentiation approach of parameter or shape sensitivity analysis [37], consists in seeking the asymptotic behavior of $\boldsymbol{v}_{a, \boldsymbol{z}}$ on $\Gamma_{\text {obs }}$ and plugging the result into (6). As previously discussed on several occasions (e.g. [13, 16]), however, a more compact and efficient formulation for the actual evaluation of $\mathbb{T}(\boldsymbol{z} ; \mathcal{S}, T)$ can be set up using an adjoint solution and is adopted here.

The adjoint formulation stems from treating the integral in the left-hand side of (6) as one of the terms arising in the reciprocity identity linking two elastodynamic states $[2,55]$, in which one state is the scattered field $\boldsymbol{v}_{a, \boldsymbol{z}}$ while the other is, like in $[13,16]$, chosen as the adjoint state $\hat{\boldsymbol{u}}$ governed by the following IBVP

$$
\begin{array}{ll}
\mathcal{L} \hat{\boldsymbol{u}}(\boldsymbol{\xi}, t)=\mathbf{0} & (\boldsymbol{\xi} \in \Omega, 0 \leq t \leq T) \\
\boldsymbol{t}[\hat{\boldsymbol{u}}](\boldsymbol{\xi}, t)=\frac{\partial \varphi}{\partial \boldsymbol{u}}[\boldsymbol{u}(\boldsymbol{\xi}, T-t), \boldsymbol{\xi}, T-t] & \left(\boldsymbol{\xi} \in \Gamma_{\mathrm{obs}}, 0 \leq t \leq T\right) \\
\boldsymbol{t}[\hat{\boldsymbol{u}}](\boldsymbol{\xi}, t)=\mathbf{0} & \left(\boldsymbol{\xi} \in \Gamma_{\mathrm{N}} \backslash \Gamma_{\mathrm{obs}}, 0 \leq t \leq T\right) \\
\hat{\boldsymbol{u}}(\boldsymbol{\xi}, t)=\mathbf{0} & \left(\boldsymbol{\xi} \in \Gamma_{\mathrm{D}}, 0 \leq t \leq T\right) \\
\hat{\boldsymbol{u}}(\boldsymbol{\xi}, 0)=\dot{\hat{\boldsymbol{u}}}(\boldsymbol{\xi}, 0)=\mathbf{0} & (\boldsymbol{\xi} \in \Omega) .
\end{array}
$$

Recall that the time convolution $\boldsymbol{a} \star \boldsymbol{b}$ of generic time-dependent tensor fields $\boldsymbol{a}, \boldsymbol{b}$ (assumed to be at rest at all negative times) is defined by

$$
[\boldsymbol{a} \star \boldsymbol{b}](\boldsymbol{\xi}, t)=\int_{0}^{t} \boldsymbol{a}(\boldsymbol{\xi}, \tau) \otimes \boldsymbol{b}(\boldsymbol{\xi}, t-\tau) \mathrm{d} \tau \quad(t \geq 0) .
$$

Moreover, the combination of time convolution and single (resp. double) inner product will be

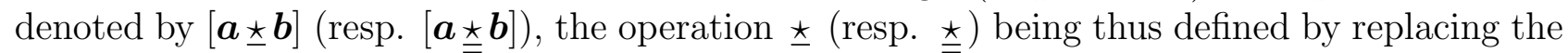
tensor product ' $\otimes$ ' sign by the inner product "." (resp. ":") sign under the integral in (8).

Now, for any generic bounded domain $\mathcal{O}$ and pair of elastodynamic states $\boldsymbol{u}_{i}(i=1,2)$ satisfying $\mathcal{L} \boldsymbol{u}_{i}=\mathbf{0}$ and initial-rest conditions $\boldsymbol{u}_{i}(\boldsymbol{\xi}, 0)=\dot{\boldsymbol{u}}_{i}(\boldsymbol{\xi}, 0)=\mathbf{0}$ in $\mathcal{O}$, the following reciprocity identity holds $[2,55]$ :

$$
\int_{\partial \mathcal{O}}\left\{\boldsymbol{t}\left[\boldsymbol{u}_{1}\right]_{\star} \boldsymbol{u}_{2}-\boldsymbol{t}\left[\boldsymbol{u}_{2}\right]_{\star} \boldsymbol{u}_{1}\right\}(\boldsymbol{\xi}, t) \mathrm{d} S_{\xi}=0 .
$$

On writing the reciprocity identity (9) for the domain $\mathcal{O}=\Omega_{a, \boldsymbol{z}}$ (with boundary $\partial \Omega_{a, \boldsymbol{z}}=\Gamma \cup S_{a, \boldsymbol{z}}$ ) and elastodynamic states $\boldsymbol{u}_{1}=\hat{\boldsymbol{u}}$ and $\boldsymbol{u}_{2}=\boldsymbol{v}_{a, \boldsymbol{z}},(6)$ is recast as

$$
\int_{S_{a, \boldsymbol{z}}}\left[\boldsymbol{t}[\hat{\boldsymbol{u}}]_{\star} \Delta \boldsymbol{v}_{a, \boldsymbol{z}}\right](\boldsymbol{\xi}, T) \mathrm{d} S_{\xi}=\eta(a) \mathbb{T}(\boldsymbol{z} ; \mathcal{S}, T)+o(\eta(a)) \quad(a \rightarrow 0),
$$

where $\Delta \boldsymbol{v}_{a, \boldsymbol{z}}:=\boldsymbol{v}_{a, \boldsymbol{z}}^{+}-\boldsymbol{v}_{a, \boldsymbol{z}}^{-}$denotes the crack opening displacement (COD), the traction vector $\boldsymbol{t}[\hat{\boldsymbol{u}}]$ is defined on $S_{a, \boldsymbol{z}}$ in terms of the unit normal $\boldsymbol{n}=\boldsymbol{n}^{-}$, and having used the boundary conditions of problems (5) and (7) and the continuity of the adjoint displacement $\hat{\boldsymbol{u}}$ across $S_{a, \boldsymbol{z}}$. Finding $\mathbb{T}(\boldsymbol{z} ; \mathcal{S}, T)$ and $\eta(a)$ from (10) now requires determining the leading behavior of the $\mathrm{COD} \Delta \boldsymbol{v}_{a, \boldsymbol{z}}$ as $a \rightarrow 0$. 


\subsection{Asymptotic analysis}

\subsubsection{Small-crack asymptotics: COD (inner expansion)}

This issue is conveniently treated by reformulating the governing IBVP (5) in terms of an integral equation, as the geometrical support of the latter is the vanishing crack surface. Let $\boldsymbol{U}(\boldsymbol{x}, t, \boldsymbol{\xi})$ and $\boldsymbol{\Sigma}(\boldsymbol{x}, t, \boldsymbol{\xi})$ denote the time-impulsive elastodynamic Green's tensors, defined so that $U_{k i}(\boldsymbol{x}, t, \boldsymbol{\xi}) \boldsymbol{e}_{i}$ and $\Sigma_{k i j}(\boldsymbol{x}, t, \boldsymbol{\xi}) \boldsymbol{e}_{i} \otimes \boldsymbol{e}_{j}$ are respectively the displacement vector and stress tensor at $\boldsymbol{\xi} \in \Omega$ and time $t$ resulting from a unit time-impulsive point force applied at $\boldsymbol{x} \in \Omega$ in the $k$-th direction at time $t=0$, satisfying the boundary conditions

$$
\boldsymbol{U}(\boldsymbol{x}, t, \boldsymbol{\xi})=\mathbf{0}\left(\boldsymbol{\xi} \in \Gamma_{\mathrm{D}}, t \geq 0\right), \quad \boldsymbol{\Sigma}(\boldsymbol{x}, t, \boldsymbol{\xi}) \cdot \boldsymbol{n}=\mathbf{0} \quad\left(\boldsymbol{\xi} \in \Gamma_{\mathrm{N}}, t \geq 0\right),
$$

and assumed to be at rest for $t \leq 0$. One also defines the elastodynamic full-space fundamental tensors $\boldsymbol{U}_{\infty}(\boldsymbol{x}, t, \boldsymbol{\xi})$ and $\boldsymbol{\Sigma}_{\infty}(\boldsymbol{x}, t, \boldsymbol{\xi})$, see Appendix B. Taking into account the homogeneous boundary conditions in (5), the COD verifies the singular integral equation [15]

$$
\begin{aligned}
& \boldsymbol{t}[\boldsymbol{u}](\boldsymbol{x}, t)=\boldsymbol{n}(\boldsymbol{x}) \cdot \mathcal{C}:\left\{\int_{S_{a, \boldsymbol{z}}} \boldsymbol{\Sigma}(\boldsymbol{x}, t, \boldsymbol{\xi}) \stackrel{\star}{=} \mathcal{D}\left[\Delta \boldsymbol{v}_{a, \boldsymbol{z}}\right](\boldsymbol{\xi}, t) \mathrm{d} S_{\xi}\right. \\
& \left.+\rho \int_{S_{a, \boldsymbol{z}}}\left[\boldsymbol{U}(\boldsymbol{x}, t, \boldsymbol{\xi}) \star \Delta \ddot{\boldsymbol{v}}_{a, \boldsymbol{z}}(\boldsymbol{\xi}, t)\right] \otimes \boldsymbol{n}(\boldsymbol{\xi}) \mathrm{d} S_{\xi}\right\} \quad\left(\boldsymbol{x} \in S_{a, \boldsymbol{z}}, 0 \leq t \leq T\right)
\end{aligned}
$$

where $\boldsymbol{w} \mapsto \mathcal{D}[\boldsymbol{w}]=\boldsymbol{e}_{i} \otimes\left(\boldsymbol{\nabla} w_{i} \otimes \boldsymbol{n}-\boldsymbol{n} \otimes \boldsymbol{\nabla} w_{i}\right)$, i.e. $\mathcal{D}[\boldsymbol{w}]_{i j k}=\left(w_{i, j} n_{k}-w_{i, k} n_{j}\right)$ in component notation, defines a (tensorial) tangential differential operator (since upon splitting the gradients into sums of tangential and normal parts, all normal derivatives cancel out), whose value hence depends only on the surface trace of $\boldsymbol{w}$. Besides, the symbol $f$ indicates a strongly singular integral defined in the Cauchy principal value sense.

The asymptotic form of integral equation (12) as $a \rightarrow 0$ is now sought. For this purpose, and following customary practice for such asymptotic analyses, scaled coordinates $\bar{\xi}$ are introduced so that

$$
\text { (a) } \boldsymbol{\xi}=\boldsymbol{z}+a \overline{\boldsymbol{\xi}}, \quad \text { (b) } \mathrm{d} S_{\xi}=a^{2} \mathrm{~d} S_{\bar{\xi}} \quad\left(\boldsymbol{\xi} \in S_{a, \boldsymbol{z}}, \overline{\boldsymbol{\xi}} \in \mathcal{S}\right)
$$

Assuming that the free field is such that $\boldsymbol{x} \mapsto \boldsymbol{\sigma}[\boldsymbol{u}](\boldsymbol{x}, t)$ is continuous at $\boldsymbol{x}=\boldsymbol{z}$, one has

$$
\boldsymbol{t}[\boldsymbol{u}](\boldsymbol{x}, t)=\boldsymbol{\sigma}[\boldsymbol{u}](\boldsymbol{z}, t) \cdot \boldsymbol{n}(\boldsymbol{x})+o(1) \quad\left(\boldsymbol{x} \in S_{a, \boldsymbol{z}}\right)
$$

Investigating the small-crack asymptotic behavior of the right-hand side of (12) is more involved, and is helped by the following lemma, whose proof is given in Appendix B:

Lemma 1. Let the vector function $\overline{\boldsymbol{v}}_{a, \boldsymbol{z}}(\overline{\boldsymbol{\xi}}, t)$ be defined by $\overline{\boldsymbol{v}}_{a, \boldsymbol{z}}(\overline{\boldsymbol{\xi}}, t)=\boldsymbol{v}_{a, \boldsymbol{z}}(\boldsymbol{\xi}, t)$, with $\overline{\boldsymbol{\xi}}$ and $\boldsymbol{\xi}$ related through (13a). Then, for $\boldsymbol{x} \in S_{a, \boldsymbol{z}}$ (i.e. $\overline{\boldsymbol{x}} \in \mathcal{S}$ ), one has

$$
\begin{aligned}
& \int_{S_{a, \boldsymbol{z}}} \boldsymbol{\Sigma}(\boldsymbol{x}, t, \boldsymbol{\xi}) \stackrel{\star}{=} \mathcal{D} \Delta \boldsymbol{v}_{a, \boldsymbol{z}}(\boldsymbol{\xi}, t) d S_{\xi} \\
& =\frac{1}{a} \int_{\mathcal{S}} \boldsymbol{\Sigma}_{\infty, a}(\overline{\boldsymbol{x}}, t, \overline{\boldsymbol{\xi}}) \stackrel{\star}{=} \mathcal{D} \Delta \overline{\boldsymbol{v}}_{a, \boldsymbol{z}}(\overline{\boldsymbol{\xi}}, t) d S_{\bar{\xi}}+O\left(a\left\|\mathcal{D}\left[\Delta \overline{\boldsymbol{v}}_{a, \boldsymbol{z}}(\cdot, t)\right]\right\|_{L^{2}(\mathcal{S})}\right) \\
& \int_{S_{a, \boldsymbol{z}}}\left[\boldsymbol{U}(\boldsymbol{x}, t, \boldsymbol{\xi}) \star \Delta \ddot{\boldsymbol{v}}_{a, \boldsymbol{z}}(\boldsymbol{\xi}, t)\right] \otimes \boldsymbol{n}(\boldsymbol{\xi}) d S_{\xi} \\
& =a \int_{\mathcal{S}}\left[\boldsymbol{U}_{\infty, a}(\overline{\boldsymbol{x}}, t, \overline{\boldsymbol{\xi}}) \star \Delta \ddot{\overline{\boldsymbol{v}}}_{a, \boldsymbol{z}}(\overline{\boldsymbol{\xi}}, t)\right] \otimes \boldsymbol{n}(\overline{\boldsymbol{\xi}}) d S_{\bar{\xi}}+O\left(a^{2}\left\|\Delta \ddot{\overline{\boldsymbol{v}}}_{a, \boldsymbol{z}}(\cdot, t)\right\|_{L^{2}(\mathcal{S})}\right)
\end{aligned}
$$

where $\boldsymbol{U}_{\infty, a}, \boldsymbol{\Sigma}_{\infty, a}$ is the full-space elastodynamic fundamental solution defined in terms of 
rescaled wave velocities $c_{L} / a, c_{T} / a$.

The left-hand side of equation (12) is $O(1)$ as $a \rightarrow 0$, with its leading contribution linear in $\boldsymbol{\sigma}[\boldsymbol{u}](\boldsymbol{z}, t)$ as seen in (14); moreover, the right-hand side of equation (12) is $O\left(a^{-1}\right)$ by Lemma 1. These remarks suggest to consider the following ansatz for $\Delta \overline{\boldsymbol{v}}_{a, \boldsymbol{z}}(\overline{\boldsymbol{\xi}}, \tau)$, in terms of unknown vector fields $\boldsymbol{V}^{i j}(\overline{\boldsymbol{\xi}})(1 \leq i, j \leq 3)$ defined on $\mathcal{S}$ :

$$
\overline{\boldsymbol{v}}_{a, \boldsymbol{z}}(\overline{\boldsymbol{\xi}}, t)=a \sigma_{i j}[\boldsymbol{u}](\boldsymbol{z}, t) \boldsymbol{V}^{i j}(\overline{\boldsymbol{\xi}})+o(a) \quad(\overline{\boldsymbol{\xi}} \in \mathcal{S})
$$

This ansatz indeed causes the first and second integrals in the right-hand side of (12) to be $O(1)$ and $o(1)$ as $a \rightarrow 0$, respectively:

Lemma 2. Assume that $\tau \mapsto \boldsymbol{\nabla} \boldsymbol{u}(\boldsymbol{\xi}, \tau)$ is twice differentiable in a neighbourhood of $\tau=t$, and let $\overline{\boldsymbol{v}}_{a, \boldsymbol{z}}(\overline{\boldsymbol{\xi}}, t)$ be of form (16) for some $\boldsymbol{V}^{i j}$. Then, for $\boldsymbol{x} \in S_{a, \boldsymbol{z}}$ (i.e. $\left.\overline{\boldsymbol{x}} \in \mathcal{S}\right)$ :

$$
f_{S_{a, \boldsymbol{z}}} \boldsymbol{\Sigma}(\boldsymbol{x}, t, \boldsymbol{\xi}) \triangleq \mathcal{D}\left[\Delta \boldsymbol{v}_{a, \boldsymbol{z}}\right](\boldsymbol{\xi}, t) d S_{\xi}=\sigma_{i j}[\boldsymbol{u}](\boldsymbol{z}, t) f_{\mathcal{S}} \boldsymbol{\Sigma}_{\infty}(\overline{\boldsymbol{\xi}}-\overline{\boldsymbol{x}}): \mathcal{D}\left[\Delta \boldsymbol{V}^{i j}\right](\overline{\boldsymbol{\xi}}) d S_{\bar{\xi}}+o(1),
$$

where $\boldsymbol{\Sigma}_{\infty}(\overline{\boldsymbol{r}})$ is the stress associated with the elastostatic Kelvin fundamental solution, given by (B.12), and

$$
\int_{S_{a, \boldsymbol{z}}}\left[\boldsymbol{U}(\boldsymbol{x}, t, \boldsymbol{\xi}) \star \Delta \ddot{\boldsymbol{v}}_{a, \boldsymbol{z}}(\boldsymbol{\xi}, t)\right] \otimes \boldsymbol{n}(\boldsymbol{\xi}) d S_{\xi}=O\left(a^{2}\right)
$$

Proof. See Appendix B.

Combining (14) with Lemma 2 allows to set up the asymptotic form of integral equation (12) in the small-crack limit (it is in particular noted that the second integral of (12) does not contribute to the limiting integral equation as its order in $a$ is higher than that of the first integral). On enforcing the limiting integral equation for any choice of $\sigma_{i j}[\boldsymbol{u}](\boldsymbol{z}, t)$, the asymptotic behavior of the COD is found to follow the representation (16), with the $\boldsymbol{V}^{i j}$ solving the integral equation

$$
\frac{1}{2}\left(\boldsymbol{e}_{i} \otimes \boldsymbol{e}_{j}+\boldsymbol{e}_{j} \otimes \boldsymbol{e}_{i}\right) \cdot \boldsymbol{n}(\overline{\boldsymbol{x}})=\boldsymbol{n}(\overline{\boldsymbol{x}}) \cdot \mathcal{C}:\left\{\int_{\mathcal{S}} \boldsymbol{\Sigma}_{\infty}(\overline{\boldsymbol{\xi}}-\overline{\boldsymbol{x}}): \mathcal{D} \Delta \boldsymbol{V}^{i j}(\overline{\boldsymbol{\xi}}) \mathrm{d} S_{\bar{\xi}}\right\} \quad(\overline{\boldsymbol{x}} \in \mathcal{S})
$$

which is readily recognised [15] as governing the exterior elastostatic problem for the crack $\mathcal{S}$ embedded in an infinite elastic medium and subjected on its faces to tractions $\boldsymbol{t}^{ \pm}=-\frac{1}{2}\left(\boldsymbol{e}_{j} \otimes\right.$ $\left.\boldsymbol{e}_{k}+\boldsymbol{e}_{k} \otimes \boldsymbol{e}_{j}\right) \cdot \boldsymbol{n}^{ \pm}(\overline{\boldsymbol{x}})$. The left-hand side of (18) being symmetric in $i, j$, there are six distinct such equations, governing six canonical solutions $\Delta \boldsymbol{V}^{i j}(\overline{\boldsymbol{\xi}})(1 \leq i \leq j \leq 3)$ which are time-independent. Like for the previously-considered small-cavity asymptotics in the time domain $[13,16]$, the functions $\Delta \boldsymbol{V}^{i j}$ depend only on the chosen (crack) shape $\mathcal{S}$; in particular, they depend neither on the shape of the solid $\Omega$ being probed, nor on the location of the sampling point $\boldsymbol{z} \in \Omega$. Finding the $\Delta \boldsymbol{V}^{i j}(\overline{\boldsymbol{\xi}})$ entails in the worst case the numerical solution of the elastostatic integral equation (18), using e.g. boundary elements, for six different sets of prescribed tractions. Analytical solutions are known for simple crack shapes (see Sec. 2.2.5). The foregoing analysis finally leads to the following result:

Proposition 1 (inner expansion). The COD on the small trial crack $S_{a, z}$ has, for any given incident stress history $\boldsymbol{\sigma}[\boldsymbol{u}]$, the asymptotic behavior

$$
\Delta \overline{\boldsymbol{v}}_{a, \boldsymbol{z}}(\overline{\boldsymbol{\xi}}, t)=a \sigma_{i j}[\boldsymbol{u}](\boldsymbol{z}, t): \Delta \boldsymbol{V}^{i j}(\overline{\boldsymbol{\xi}})+o(a) \quad(\overline{\boldsymbol{\xi}} \in \mathcal{S}),
$$

where $\Delta \boldsymbol{V}^{i j}$ solves the elastostatic integral equation (18) for fixed $(i, j), 1 \leq i \leq j \leq 3$. 


\subsubsection{Small-crack asymptotics (outer expansion)}

The scattered displacement at any location $\boldsymbol{x} \in \Omega_{a, \boldsymbol{z}}$, i.e. away from the trial crack $S_{a, \boldsymbol{z}}$, is then explicitly given by the integral representation formula [15]

$$
\boldsymbol{v}_{a, \boldsymbol{z}}(\boldsymbol{x}, t)=\int_{S_{a, \boldsymbol{z}}}[\boldsymbol{\Sigma}(\boldsymbol{x}, t, \boldsymbol{\xi}) \cdot \boldsymbol{n}(\boldsymbol{\xi})]_{\underline{\Delta}} \Delta \boldsymbol{v}_{a, \boldsymbol{z}}(\boldsymbol{\xi}, t) \mathrm{d} S_{\xi} \quad \boldsymbol{x} \in \Omega_{a, \boldsymbol{z}}
$$

Finding the small-crack asymptotics of (20) is helped by the following lemma:

Lemma 3. Let the function $\tau \mapsto f(\tau)$ be defined and twice differentiable in a neighbourhood of $\tau=t$. For any $\boldsymbol{x} \in \Omega, \boldsymbol{x} \neq \boldsymbol{z}$, and for any a sufficiently small (i.e. such that $\boldsymbol{x} \notin \bar{S}_{a, \boldsymbol{z}}$ ), one has

$$
\boldsymbol{\Sigma}(\boldsymbol{x}, t, \boldsymbol{\xi}) \star f(t)=\boldsymbol{\Sigma}(\boldsymbol{z}, t, \boldsymbol{\xi}) \star f(t)+O(a) \quad(a \rightarrow 0)
$$

Proof. See Appendix B.

Inserting expansion (19) into (20), using the coordinate scaling (13) and invoking Lemma 3 with $f(t)=\sigma_{i j}(\boldsymbol{z}, t)$, one arrives at the following expansion for $\boldsymbol{v}_{a, \boldsymbol{z}}$ away from the crack (outer expansion).

Proposition 2 (outer expansion). Let the time-independent CODs $\Delta \boldsymbol{V}^{i j}$, which solve the elastostatic integral equation (18) for each fixed $(i, j), 1 \leq i \leq j \leq 3$, be arranged into a thirdorder tensor field $\Delta \mathcal{V}(\overline{\boldsymbol{\xi}})$ such that $\Delta \boldsymbol{V}^{i j}=\Delta \mathcal{V}_{i j k} \boldsymbol{e}_{k}$. Define the fourth-order elastic moment tensors $\mathcal{A}^{\sigma}$ and $\mathcal{A}$ by

$$
\mathcal{A}^{\sigma}(\mathcal{S})=\int_{\mathcal{S}} \Delta \mathcal{V}(\overline{\boldsymbol{\xi}}) \otimes \boldsymbol{n}(\overline{\boldsymbol{\xi}}) d S_{\bar{\xi}}, \quad \mathcal{A}(\mathcal{S})=\mathcal{C}: \mathcal{A}^{\sigma}(\mathcal{S}): \mathcal{C}
$$

Then, the scattered field $\boldsymbol{v}_{a, \boldsymbol{z}}$ evaluated at any location $\boldsymbol{x} \in \Omega, \boldsymbol{x} \neq \boldsymbol{z}$ has, for any a small enough to have $\boldsymbol{x} \notin \bar{S}_{a, \boldsymbol{z}}$, the asymptotic behavior

$$
\begin{aligned}
& \boldsymbol{v}_{a, \boldsymbol{z}}(\boldsymbol{x}, t)=a^{3} \boldsymbol{\Sigma}(\boldsymbol{x}, t, \boldsymbol{z}) \stackrel{\star}{=}\left[\mathcal{A}^{\sigma}(\mathcal{S}): \boldsymbol{\sigma}[\boldsymbol{u}](\boldsymbol{z}, t)\right]+o\left(a^{3}\right) \\
& =a^{3} \boldsymbol{\nabla}_{z} \boldsymbol{U}(\boldsymbol{x}, t, \boldsymbol{z}) \stackrel{\star}{=}[\mathcal{A}(\mathcal{S}): \boldsymbol{\nabla} \boldsymbol{u}(\boldsymbol{z}, t)]+o\left(a^{3}\right)
\end{aligned}
$$

Lemma 4. The elastic moment tensor $\mathcal{A}^{\sigma}$ defined in Proposition 2 has major symmetry: for any second-order symmetric tensors $\boldsymbol{\sigma}, \boldsymbol{\sigma}^{\prime} \in \mathbb{R}_{\text {sym }}^{3,3}$, one has

$$
\sigma: \mathcal{A}^{\sigma}: \sigma^{\prime}=\sigma^{\prime}: \mathcal{A}^{\sigma}: \sigma
$$

Proof. See Appendix A.1.

Remark 1. The elastic moment tensor depends only on the crack shape $\mathcal{S}$ and the elastic properties of the reference medium. It can be computed (at least numerically) for any assumed shape $\mathcal{S}$, while analytical solutions are available for simple shapes of $\mathcal{S}$ (e.g. penny-shaped or elliptical cracks, see Sec. 2.2.5).

Remark 2. The symmetry property (24) also holds for elastic moment tensors arising in connection with elastic inhomogeneities, see e.g. $[8,18]$, and is the elastic counterpart of the well-known symmetry of polarization tensors for conductivity heterogeneities [25]. 


\subsubsection{Topological derivative}

Now, inserting expansion (19) into the left-hand side of (10) and invoking (13b), one obtains

$$
\begin{aligned}
\int_{0}^{T} \int_{S_{a, \boldsymbol{z}}} \boldsymbol{t}[\hat{\boldsymbol{u}}](\boldsymbol{\xi}, T & -t) \cdot \Delta \boldsymbol{v}_{a, \boldsymbol{z}}(\boldsymbol{\xi}, t) \mathrm{d} S_{\xi} \mathrm{d} t \\
& =a^{3} \int_{0}^{T} \int_{\mathcal{S}} \boldsymbol{\sigma}[\boldsymbol{u}](\overline{\boldsymbol{\xi}}, t):(\Delta \mathcal{V}(\overline{\boldsymbol{\xi}}) \otimes \boldsymbol{n}(\overline{\boldsymbol{\xi}})): \boldsymbol{\sigma}[\hat{\boldsymbol{u}}](\overline{\boldsymbol{\xi}}, T-t) \mathrm{d} S_{\bar{\xi}} \mathrm{d} t+o\left(a^{3}\right) .
\end{aligned}
$$

A comparison of $(25)$ with $(10)$ then readily allows to identify $\mathbb{T}(\boldsymbol{z} ; \mathcal{S}, T)$ and $\eta(a)$, leading to the main result of this section:

Theorem 1 (adjoint-based formulation of topological derivative). The topological derivative $\mathbb{T}(\boldsymbol{z} ; \mathcal{S}, T)$ of $\mathbb{J}$ and its small-crack asymptotic behavior $\eta(a)$ are given by

$$
\begin{aligned}
\mathbb{T}(\boldsymbol{z} ; \mathcal{S}, T) & =\left[\boldsymbol{\sigma}[\boldsymbol{u}]_{\underline{太}}\left(\mathcal{A}^{\sigma}(\mathcal{S}): \boldsymbol{\sigma}[\hat{\boldsymbol{u}}]\right)\right](\boldsymbol{z}, T)=[\boldsymbol{\nabla} \boldsymbol{u} \underset{\underline{\underline{A}}}{=}(\mathcal{A}(\mathcal{S}): \nabla \hat{\boldsymbol{u}})](\boldsymbol{z}, T), \\
\eta(a) & =a^{3},
\end{aligned}
$$

in terms of the free and adjoint fields $\boldsymbol{u}$ and $\hat{\boldsymbol{u}}$, and with the elastic moment tensors $\mathcal{A}^{\sigma}, \mathcal{A}$ defined by (22).

Remark 3. The major symmetry of $\mathcal{A}^{\sigma}$ (Lemma 4), combined with the major and minor symmetries of $\mathcal{C}$, implies

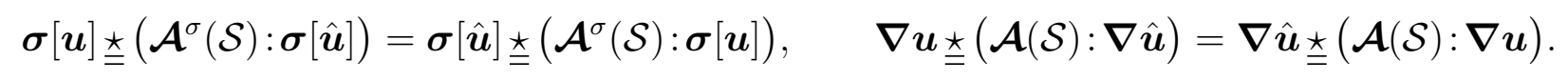

Remark 4. The topological derivative (26a) depends on the assumed trial crack shape $\mathcal{S}$ through $\mathcal{A}(\mathcal{S})$.

Remark 5. An alternative formulation of the topological derivative, equivalent to (26a), could have been obtained by introducing the outer expansion (23) into (6), a manipulation which also reveals at once the $O\left(a^{3}\right)$ leading behavior of $\mathbb{J}\left(\Omega_{a, \boldsymbol{z}}, T\right)$. The adjoint solution-based form (26a) of $\mathbb{T}(\boldsymbol{z} ; \mathcal{S}, T)$, which is deemed preferable here because of its greater simplicity and ease of implementation, does not require (23) for its establishment. In fact, the latter can be obtained as a special case of (26a) by considering the cost function $\mathbb{J}\left(\Omega_{a, \boldsymbol{z}}, T\right):=\boldsymbol{v}_{a, \boldsymbol{z}}(\boldsymbol{x}, T)$, for which the adjoint solution $\hat{\boldsymbol{u}}$ reduces to the time-reversed version of the Green's tensor $\boldsymbol{U}(\boldsymbol{x}, t, \boldsymbol{\xi})$.

Remark 6. The $O\left(a^{3}\right)$ asymptotic behavior (26ab) relies on $\boldsymbol{v}_{a, \boldsymbol{z}}$ approaching (up to a scaling factor) a static solution as $a \rightarrow 0$. This requires the free-field to be sufficiently regular at $(\boldsymbol{z}, t)$, e.g. according to the sufficient condition given in Lemma 2, which induces a limitation of the high-frequency content of the excitation (see also [3]).

\subsubsection{An alternative expression for the elastic moment tensor}

The elastic moment tensor $\mathcal{A}^{\sigma}$ for given normalized trial crack $\mathcal{S}$ can be evaluated directly from (22) using either analytical or numerical solutions for $\Delta \mathcal{V}$. The former are available only for quite simple crack shapes, including the penny-shaped crack (see Sec. 2.2.5) and the elliptical crack (see [42]). Numerical evaluation of $\mathcal{A}^{\sigma}$ may otherwise be achieved from solving the elastostatic normalized integral equation (18) using the boundary element method. In addition, an alternative evaluation method for $\mathcal{A}^{\sigma}$, relying on the knowledge of the stress intensity factors (SIFs) associated with $\Delta \mathcal{V}$, rather than $\Delta \mathcal{V}$ itself, is given next in Proposition 3; it may be convenient in some cases because many analytical or semi-analytical solutions for linear fracture 
mechanics problems are available in terms of their SIFs (see compendia such as [53]) rather than their COD.

Proposition 3. The elastic moment tensor $\mathcal{A}^{\sigma}(\mathcal{S})$ satisfies

$$
\boldsymbol{\sigma}: \mathcal{A}^{\sigma}(\mathcal{S}): \boldsymbol{\sigma}=\frac{2}{3} \int_{\partial \mathcal{S}} G(s)(\boldsymbol{\nu}(s) \cdot \boldsymbol{\xi}(s)) d s
$$

where $\boldsymbol{\sigma} \in \mathbb{R}_{\text {sym }}^{3,3}$ is any constant symmetric tensor, $G(s)$ is the energy release rate associated to the $C O D \boldsymbol{\sigma}: \Delta \mathcal{V}$ at a point of the crack front $\partial \mathcal{S}$ (parameterized by the arc length $s$ ), and $\boldsymbol{\nu}(s$ ) is the outward unit normal to $\partial \mathcal{S}$ lying in the tangent plane of $\mathcal{S}$. If the reference medium is isotropic, applying Irwin's formula [53] to (27) yields the more explicit expression

$$
\boldsymbol{\sigma}: \mathcal{A}^{\sigma}(\mathcal{S}): \boldsymbol{\sigma}=\frac{1}{3 \mu} \int_{\partial \mathcal{S}}\left[(1-\nu)\left(K_{I}^{2}(s)+K_{I I}^{2}(s)\right)+K_{I I I}^{2}(s)\right](\boldsymbol{\nu}(s) \cdot \boldsymbol{\xi}(s)) d s
$$

where $K_{I}(s), K_{I I}(s), K_{I I I}(s)$ are the stress intensity factors associated to the COD $\boldsymbol{\sigma}: \Delta \mathcal{V}$.

Proof. See Appendix A.2.

\subsubsection{Elastic moment tensor for elliptic or circular cracks}

Proposition 3 is now applied to the case of a trial crack of elliptical (or circular) shape. Let $\mathcal{S}$ denote an elliptical crack whose major semiaxis $\ell_{1}$ and minor semiaxis $\ell_{2}=q^{\prime} \ell_{1}\left(\right.$ with $\left.0<q^{\prime}<1\right)$ are respectively aligned with the $\boldsymbol{e}_{1}$ and $\boldsymbol{e}_{2}$ directions of a Cartesian frame (the normal $\boldsymbol{n}$ to $\mathcal{S}$ thus being $\boldsymbol{n}=\boldsymbol{e}_{3}$ ). An analytical solution is available for both the COD and the SIFs [42]. The SIFs, which for this problem have simpler analytical expressions than the COD, are given at any point of $\partial \mathcal{S}$ by

$$
\begin{aligned}
K_{\mathrm{I}}=\frac{\sigma_{33} \sqrt{\pi q^{\prime} \ell_{1}} \Delta^{1 / 4}(\phi)}{E(q)}, \quad K_{\mathrm{II}} & =-\frac{q^{2} \sqrt{\pi q^{\prime} \ell_{1}}}{\Delta^{1 / 4}(\phi)}\left[\frac{\sigma_{23}}{D_{23}(q)} \sin \phi+q^{\prime} \frac{\sigma_{13}}{D_{13}(q)} \cos \phi\right], \\
K_{\mathrm{III}} & =(1-\nu) \frac{q^{2} \sqrt{\pi q^{\prime} \ell_{1}}}{\Delta^{1 / 4}(\phi)}\left[\frac{\sigma_{13}}{D_{13}(q)} \sin \phi-q^{\prime} \frac{\sigma_{23}}{D_{23}(q)} \cos \phi\right],
\end{aligned}
$$

where $\phi \in\left[0,2 \pi\left[\right.\right.$ is the angle in the parametric representation $\left(\xi_{1}, \xi_{2}\right)=\ell_{1}\left(\cos \phi, q^{\prime} \sin \phi\right)$ of $\partial \mathcal{S}$, $\Delta(\phi)=1-q^{2} \cos ^{2} \phi\left(\right.$ where $\left.q=\left(1-q^{\prime 2}\right)^{1 / 2}\right)$, and the constants $D_{13}, D_{23}$ are given by

$$
D_{13}(q)=\left(q^{2}-\nu\right) E(q)+\nu q^{\prime 2} K(q), \quad D_{23}(q)=\left(q^{2}+\nu q^{2}\right) E(q)-\nu q^{2} K(q)
$$

with $K(q), E(q)$ denoting the complete elliptic integrals of the first and second kind, respectively [1]:

$$
K(q)=\int_{0}^{\pi / 2}\left(1-q^{2} \sin ^{2} \phi\right)^{-1 / 2} \mathrm{~d} \phi, \quad E(q)=\int_{0}^{\pi / 2}\left(1-q^{2} \sin ^{2} \phi\right)^{1 / 2} \mathrm{~d} \phi .
$$

Substituting expressions (29) into (28), noting that the chosen parametric representation of $\partial \mathcal{S}$ implies that $(\boldsymbol{\nu}(s) \cdot \boldsymbol{\xi}(s)) \mathrm{d} s=q^{\prime} \ell_{1}^{2} \mathrm{~d} \phi$ and performing the resulting integral with the help of formulae $2.584(4,6)$ of [34], $\mathcal{A}^{\sigma}$ is obtained as

$$
\begin{aligned}
\mathcal{A}^{\sigma}=\frac{\pi(1-\nu) q^{\prime 2} \ell_{1}^{3}}{3 \mu} & {\left[\frac{q^{2}}{D_{13}(q)}\left(\boldsymbol{n} \otimes \boldsymbol{e}_{1}+\boldsymbol{e}_{1} \otimes \boldsymbol{n}\right) \otimes\left(\boldsymbol{n} \otimes \boldsymbol{e}_{1}+\boldsymbol{e}_{1} \otimes \boldsymbol{n}\right)\right.} \\
+ & \left.\frac{q^{2}}{D_{23}(q)}\left(\boldsymbol{n} \otimes \boldsymbol{e}_{2}+\boldsymbol{e}_{2} \otimes \boldsymbol{n}\right) \otimes\left(\boldsymbol{n} \otimes \boldsymbol{e}_{2}+\boldsymbol{e}_{2} \otimes \boldsymbol{n}\right)+\frac{1}{E(q)} \boldsymbol{n} \otimes \boldsymbol{n} \otimes \boldsymbol{n} \otimes \boldsymbol{n}\right]
\end{aligned}
$$


Expression (32) can in particular be applied to the special case of a unit penny-shaped crack (for which $\ell_{1}=1, q=0$ and $q^{\prime}=1$ ). Inserting the expansions [1]

$$
K(q)=\frac{\pi}{2}\left[1+\frac{q^{2}}{4}\right]+o\left(q^{2}\right), \quad E(q)=\frac{\pi}{2}\left[1-\frac{q^{2}}{4}\right]+o\left(q^{2}\right) . \quad q \rightarrow 0
$$

into (30), one obtains $D_{13}(q)=D_{23}(q)=\pi(2-\nu) q^{2} / 4+o\left(q^{2}\right)$. Substituting these expansions into (32), one readily finds

$$
\mathcal{A}^{\sigma}=\frac{4(1-\nu)}{3 \mu(2-\nu)}\left[\left(\boldsymbol{n} \otimes \boldsymbol{e}_{\alpha}+\boldsymbol{e}_{\alpha} \otimes \boldsymbol{n}\right) \otimes\left(\boldsymbol{n} \otimes \boldsymbol{e}_{\alpha}+\boldsymbol{e}_{\alpha} \otimes \boldsymbol{n}\right)+2(2-\nu) \boldsymbol{n} \otimes \boldsymbol{n} \otimes \boldsymbol{n} \otimes \boldsymbol{n}\right],
$$

where $\alpha \in\{1,2\}$. Then, using (1), (22) and (33), one finds

$$
\begin{gathered}
\mathcal{A}=\frac{8 \mu(1-\nu)}{3}\left\{\left(\frac{2 \nu}{1-2 \nu}\right)^{2} \boldsymbol{I} \otimes \boldsymbol{I}+\frac{4 \nu}{1-2 \nu}[\boldsymbol{n} \otimes \boldsymbol{n} \otimes \boldsymbol{I}+\boldsymbol{I} \otimes \boldsymbol{n} \otimes \boldsymbol{n}]+4 \boldsymbol{n} \otimes \boldsymbol{n} \otimes \boldsymbol{n} \otimes \boldsymbol{n}\right. \\
\left.+\frac{2}{(2-\nu)}\left[\boldsymbol{n} \otimes \boldsymbol{e}_{\alpha}+\boldsymbol{e}_{\alpha} \otimes \boldsymbol{n}\right] \otimes\left[\boldsymbol{n} \otimes \boldsymbol{e}_{\alpha}+\boldsymbol{e}_{\alpha} \otimes \boldsymbol{n}\right]\right\} .
\end{gathered}
$$

Expression (33) can also be found by using the available [26,41] closed form solutions for the penny-shaped crack under uniform remote loading, given by

$$
\Delta \boldsymbol{V}^{i j}(\overline{\boldsymbol{\xi}})=\frac{2(1-\nu)}{\pi \mu(2-\nu)} \sqrt{1-|\overline{\boldsymbol{\xi}}|^{2}} \boldsymbol{n} \cdot\left(\boldsymbol{e}_{i} \otimes \boldsymbol{e}_{j}+\boldsymbol{e}_{j} \otimes \boldsymbol{e}_{i}\right) \cdot[2 \boldsymbol{I}-\nu \boldsymbol{n} \otimes \boldsymbol{n}] \quad(\overline{\boldsymbol{\xi}} \in \mathcal{S})
$$

in (22), performing the resulting integral and symmetrizing the resulting fourth-order tensor with respect to the minor symmetries.

\section{ACOUSTIC TOPOLOGICAL DERIVATIVE}

For completeness, the case where the reference medium is an acoustic fluid characterized by the wave velocity $c$ is now treated. In this context, a "crack" supporting homogeneous Neumann conditions models a thin rigid screen across which the acoustic pressure may be discontinuous. The governing IBVP for the scattered acoustic pressure field $v_{a, z}$ arising due to the presence of a screen $S_{a, \boldsymbol{z}}$ of small size $a$ and shape $\mathcal{S}$ located at $\boldsymbol{z}$ is:

$$
\begin{array}{ll}
c^{2} \nabla^{2} v_{a, \boldsymbol{z}}(\boldsymbol{\xi}, t)-\ddot{v}_{a, \boldsymbol{z}}(\boldsymbol{\xi}, t)=0 & \left(\boldsymbol{\xi} \in \Omega_{a, \boldsymbol{z}}, t \geq 0\right) \\
q^{ \pm}\left[v_{a, \boldsymbol{z}}^{ \pm}\right]=-q^{ \pm}[u](\boldsymbol{\xi}, t) & \left(\boldsymbol{\xi} \in S_{a, \boldsymbol{z}}^{ \pm}, t \geq 0\right) \\
q\left[v_{a, \boldsymbol{z}}\right]=0 & \left(\boldsymbol{\xi} \in \Gamma_{\mathrm{N}}, t \geq 0\right) \\
v_{a, \boldsymbol{z}}(\boldsymbol{\xi}, t)=0 & \left(\boldsymbol{\xi} \in \Gamma_{\mathrm{D}}, t \geq 0\right) \\
v_{a, \boldsymbol{z}}(\boldsymbol{\xi}, 0)=\dot{v}_{a, \boldsymbol{z}}(\boldsymbol{\xi}, 0)=0 & \left(\boldsymbol{\xi} \in \Omega_{a, \boldsymbol{z}}\right),
\end{array}
$$

where $u$ is the free field, $w \mapsto q[w]:=\boldsymbol{\nabla} w \cdot \boldsymbol{n}$ is the normal derivative operator (with the superscript \pm referring where necessary to the relevant crack face and its unit normal). Then is the same fashion as in equation (6) the topological derivative is defined for scalar waves through

$$
\int_{0}^{T} \int_{\Gamma_{\mathrm{obs}}} \frac{\partial \varphi}{\partial u}[u(\boldsymbol{\xi}, t), \boldsymbol{\xi}, t] v_{a, \boldsymbol{z}}(\boldsymbol{\xi}, t) \mathrm{d} S_{\xi} \mathrm{d} t=\eta(a) \mathbb{T}(\boldsymbol{z} ; \mathcal{S}, T)+o(\eta(a)) \quad(a \rightarrow 0) .
$$


Introducing the adjoint field $\hat{u}$ solution of the IBVP

$$
\begin{array}{ll}
c^{2} \nabla^{2} \hat{u}(\boldsymbol{\xi}, t)-\ddot{\hat{u}}(\boldsymbol{\xi}, t)=0 & (\boldsymbol{\xi} \in \Omega, 0 \leq t \leq T) \\
q[\hat{u}]=\frac{\partial \varphi}{\partial u}[u(\boldsymbol{\xi}, T-t), \boldsymbol{\xi}, T-t] & \left(\boldsymbol{\xi} \in \Gamma_{\text {obs }}, 0 \leq t \leq T\right) \\
q[\hat{u}]=0 & \left(\boldsymbol{\xi} \in \Gamma_{\mathrm{N}} \backslash \Gamma_{\text {obs }}, 0 \leq t \leq T\right) \\
\hat{u}(\boldsymbol{\xi}, t)=0 & \left(\boldsymbol{\xi} \in \Gamma_{\mathrm{D}}, 0 \leq t \leq T\right) \\
\hat{u}(\boldsymbol{\xi}, 0)=\dot{\hat{u}}(\boldsymbol{\xi}, 0)=0 & (\boldsymbol{\xi} \in \Omega),
\end{array}
$$

and invoking the scalar dynamical reciprocity identity for states $v_{a, \boldsymbol{z}}$ and $\hat{u}$, equation (35) reduces to

$$
\eta(a) \mathbb{T}(\boldsymbol{z} ; \mathcal{S}, T)+o(\eta(a))=\int_{S_{a, \boldsymbol{z}}}\left[q[\hat{u}] \star \Delta v_{a, \boldsymbol{z}}\right](\boldsymbol{\xi}, t) \mathrm{d} S_{\xi} \quad(a \rightarrow 0),
$$

where $\Delta v_{a, \boldsymbol{z}}=v_{a, \boldsymbol{z}}^{+}-v_{a, \boldsymbol{z}}^{-}$denotes the acoustic pressure jump through the screen and the flux $q[\hat{u}]$ is defined on $S_{a, \boldsymbol{z}}$ in terms of the unit normal $\boldsymbol{n}=\boldsymbol{n}^{-}$

Leading contributions as $a \rightarrow 0$. To determine the leading contributions of $v_{a, \boldsymbol{z}}$ in the limit $a \rightarrow 0$, it is convenient to reformulate the scattering IBVP (34) as a singular boundary integral equation [15]:

$$
\begin{aligned}
q[u](\boldsymbol{x}, t)=\boldsymbol{n}(\boldsymbol{x}) \cdot\{ & \int_{S_{a, \boldsymbol{z}}} \boldsymbol{\nabla} G(\boldsymbol{x}, t, \boldsymbol{\xi}) \star \mathcal{D}\left[\Delta v_{a, \boldsymbol{z}}\right](\boldsymbol{\xi}, t) \mathrm{d} S_{\xi} \\
& \left.+\frac{1}{c^{2}} \int_{S_{a, \boldsymbol{z}}} \boldsymbol{n}(\boldsymbol{\xi}) G(\boldsymbol{x}, t, \boldsymbol{\xi}) \star \Delta \ddot{v}_{a, \boldsymbol{z}}(\boldsymbol{\xi}, t) \mathrm{d} S_{\xi}\right\} \quad\left(\boldsymbol{x} \in S_{a, \boldsymbol{z}}, 0 \leq t \leq T\right)
\end{aligned}
$$

with the tangential differential operator $\mathcal{D}$ defined by $w \mapsto \mathcal{D} w=\boldsymbol{\nabla} w \otimes \boldsymbol{n}-\boldsymbol{n} \otimes \boldsymbol{\nabla} w$ and where $G(\boldsymbol{x}, t, \boldsymbol{\xi})$ is the acoustic Green's function, i.e. the pressure at $\boldsymbol{\xi}$ and time $t$ created in $\Omega$ by a time-impulsive point source acting at $\boldsymbol{x} \in \Omega$ and $t=0$ and satisfying the boundary conditions

$$
G(\boldsymbol{x}, t, \boldsymbol{\xi})=0 \quad\left(\boldsymbol{\xi}=\Gamma_{\mathrm{D}}, t \geq 0\right), \quad q[G](\boldsymbol{x}, t, \boldsymbol{\xi})=0 \quad\left(\boldsymbol{\xi}=\Gamma_{\mathrm{N}}, t \geq 0\right)
$$

Proceeding along the same lines as in Sec. 2.2.1, one arrives at a representation of $v_{a, z}$ of the form

$$
\Delta v_{a, \boldsymbol{z}}(\boldsymbol{\xi}, t)=a \frac{\partial u}{\partial z_{i}}(\boldsymbol{z}, t) \Delta V^{i}(\overline{\boldsymbol{\xi}})+o(a)
$$

and at the following expansions, which are the acoustic counterparts of $(17 a, b)$ :

$$
\begin{aligned}
& \int_{S_{a, \boldsymbol{z}}} \boldsymbol{\nabla} G(\boldsymbol{x}, t, \boldsymbol{\xi}) \star \mathcal{D}\left[\Delta v_{a, \boldsymbol{z}}\right](\boldsymbol{\xi}, t) \mathrm{d} S_{\xi}=\frac{\partial u}{\partial z_{i}}(\boldsymbol{z}, t)\left\{f_{\mathcal{S}} \nabla G_{\infty}(\overline{\boldsymbol{\xi}}-\overline{\boldsymbol{x}}) \cdot \mathcal{D} \Delta V^{i}(\overline{\boldsymbol{\xi}}) \mathrm{d} S_{\bar{\xi}}+o(1)\right\} \\
& \int_{S_{a, \boldsymbol{z}}} \boldsymbol{n}(\boldsymbol{\xi}) G(\boldsymbol{x}, t, \boldsymbol{\xi}) \star \Delta \ddot{v}_{a, \boldsymbol{z}}(\boldsymbol{\xi}, t) \mathrm{d} S_{\xi}=O\left(a^{2}\right)
\end{aligned}
$$

(with $G_{\infty}(\boldsymbol{r})=1 / 4 \pi\|\boldsymbol{r}\|$ denoting the full-space Laplace fundamental solution). Thus, deriving the limiting form of integral equation (38) as $a \rightarrow 0$ and enforcing the result for any value of $\partial u / \partial z_{i}(\boldsymbol{z}, t)$ yields governing integral equations for the $\Delta V^{i}$ :

$$
\boldsymbol{n}(\overline{\boldsymbol{x}}) \cdot \boldsymbol{e}_{i}=\boldsymbol{n}(\overline{\boldsymbol{x}}) \cdot f_{\mathcal{S}} \boldsymbol{\nabla} G_{\infty}(\overline{\boldsymbol{\xi}}-\overline{\boldsymbol{x}}) \cdot \mathcal{D} \Delta V^{i}(\overline{\boldsymbol{\xi}}) \mathrm{d} S_{\bar{\xi}} \quad(\overline{\boldsymbol{x}} \in \mathcal{S})
$$


which correspond to exterior Laplace problems for the normalized screen $\mathcal{S}$ whose faces are subjected to fluxes $q^{ \pm}(\overline{\boldsymbol{x}})=-\boldsymbol{n}^{ \pm}(\overline{\boldsymbol{x}}) \cdot \boldsymbol{e}_{i}$.

Topological derivative. Inserting (19) into the right-hand side of (37), one finally obtains

$$
\text { (a) } \mathbb{T}(\boldsymbol{z} ; \mathcal{S}, T)=[\boldsymbol{\nabla} \hat{u} \underline{\underline{ }}(\boldsymbol{B}(\mathcal{S}) \cdot \boldsymbol{\nabla} u)](\boldsymbol{z}, T), \quad \text { (b) } \eta(a)=a^{3},
$$

with the second-order polarization tensor $\mathcal{B}$ defined, upon arranging the $V^{i}$ into a vector field $\mathcal{V}=V^{i} \boldsymbol{e}_{i}$, by

$$
\boldsymbol{B}(\mathcal{S})=\int_{\mathcal{S}} \Delta \mathcal{V}(\overline{\boldsymbol{\xi}}) \otimes \boldsymbol{n}(\overline{\boldsymbol{\xi}}) \mathrm{d} S_{\bar{\xi}}
$$

An argument similar to that of Appendix A.1 readily shows that the polarization tensor $\mathcal{B}$ is symmetric.

Polarization tensor for the elliptic screen. The polarization tensor for an elliptic soundhard plane screen has the following closed-form expression:

$$
\mathcal{B}=\frac{4 \pi q^{\prime}}{3 E(q)} \ell_{1}^{3} \boldsymbol{n} \otimes \boldsymbol{n}
$$

with $\ell_{1}, q, q^{\prime}$ as defined in Sec. 2.2.5 and $E(q)$ again given by (31). This result readily follows from using the known solution [50] to problem (39), given for any $\boldsymbol{q} \in \mathbb{R}^{3}$ by

$$
\boldsymbol{q} \cdot \Delta \mathcal{V}(\overline{\boldsymbol{\xi}})=\frac{2 \ell_{1} q^{\prime}}{E(q)}\left(1-\frac{\bar{\xi}_{1}^{2}}{\ell_{1}^{2}}-\frac{\bar{\xi}_{2}^{2}}{\ell_{2}^{2}}\right)^{1 / 2} \boldsymbol{q} \cdot \boldsymbol{n}
$$

in (41) and performing analytically the resulting integral. The result (42) may alternatively be found as the limiting case for $\ell_{3} / \ell_{1} \rightarrow 0$ of the polarization tensor for a hard ellipsoidal inclusion [35].

The case of the circular screen of unit radius then corresponds to $q=0, \ell_{1}=1$ and $E(q)=\pi / 2$, i.e.:

$$
\mathcal{B}=\frac{8}{3} \boldsymbol{n} \otimes \boldsymbol{n}
$$

\section{TD-BASED CRACK IDENTIFICATION: HEURISTICS AND IMPLEMEN- TATION}

Topological derivative as a crack indicator function. Since $\mathbb{T}(\boldsymbol{z} ; \mathcal{S}, T)$ quantifies the sensitivity of the featured cost functional $\mathbb{J}$ to the appearance at $\boldsymbol{z}$ of an infinitesimal crack in the reference medium, it is natural to consider $\boldsymbol{z} \mapsto \mathbb{T}(\boldsymbol{z} ; \mathcal{S}, T)$ as a possible crack indicator function. This heuristic approach consists in seeking actual crack(s) at locations $\boldsymbol{z}$ at which $\mathbb{T}(\boldsymbol{z} ; \mathcal{S}, T)$ attains its most pronounced negative values (i.e. such that infinitesimal trial cracks placed there improve the fit between predicted and actual measurements). A simple shape (usually circular) will be assumed for $\mathcal{S}$, with its orientation chosen so as to minimize $\mathbb{T}(\boldsymbol{z} ; \mathcal{S}, T)$ as explained later in this section. While intuition (and previous studies on the same approach carried out for other types of defects) suggests that finite defects having the same location also induce a decrease of the cost function, this proposed exploitation of the TD field $\mathbb{T}(\cdot, \mathcal{S}, T)$ is not backed by a rigorous mathematical proof (whereas the analysis of the cost function leading to the definition and evaluation of $\mathbb{T}(\boldsymbol{z} ; \mathcal{S}, T)$ is itself mathematically rigorous). This proposed heuristic identification approach, whose main features are discussed in the remainder of this section, will be tested on numerical experiments in Sec. 5 . 
Evaluation of the topological derivative field. Computing the field $\mathbb{T}(\cdot, \mathcal{S}, T)$ using (26a) entails the evaluation of a time-convolution integral. Performing the latter operation requires storing the entire histories for the free and adjoint fields (which may be discarded once the convolution is computed). To hold this memory space over the shortest possible time in the course of the computation, it is useful to recast $\mathbb{T}(\boldsymbol{z} ; \mathcal{S}, T)$ into the equivalent form

$$
\mathbb{T}(\boldsymbol{z} ; \mathcal{S}, T)=\Pi(\boldsymbol{z}, T):: \mathcal{A}^{\sigma}(\mathcal{S})
$$

(with "::" denoting the four-fold inner product between tensors) where the fourth-order tensor field $\boldsymbol{\Pi}(\boldsymbol{z}, T)$ is defined (taking advantage of the known major symmetry of $\mathcal{A}^{\sigma}$, see Lemma 4 ) by

$$
\Pi(\boldsymbol{z}, T)=(\boldsymbol{\sigma}[\boldsymbol{u}] \star \boldsymbol{\sigma}[\hat{\boldsymbol{u}}])(\boldsymbol{z}, T)
$$

The computational advantage of (44) over (26a) is materialized by evaluating $\boldsymbol{\Pi}(\boldsymbol{z}, T)$ immediately after solving the free and adjoint IBVPs, after which the discretized free and adjoint solutions are no longer needed and may be discarded from the memory. This treatment has at least two advantages. First, when the featured cost function additively aggregates several experiments, the tensor field $\boldsymbol{\Pi}^{(k)}(\boldsymbol{z}, T)$ associated with the $k$-th experiment can be computed sequentially and accumulated onto $\boldsymbol{\Pi}(\boldsymbol{z}, T)$, so that the topological derivative for the whole set of $K$ experiments is still given by (44) but with (45) replaced by

$$
\boldsymbol{\Pi}(\boldsymbol{z}, T)=\sum_{k=1}^{K} \boldsymbol{\Pi}^{(k)}(\boldsymbol{z}, T) .
$$

The computational work for setting up $\boldsymbol{\Pi}(\boldsymbol{x}, T)$ is proportional to $K$, but that used in the subsequent evaluation of (44) is independent on $K$; moreover, only one pair of free and adjoint space-time fields needs to be stored at any time. Second, $\boldsymbol{\Pi}(\boldsymbol{z}, T)$ does not depend on the shape or orientation of $\mathcal{S}$, which affect only the constant elastic momemt tensor $\mathcal{A}^{\sigma}(\mathcal{S})$. This makes it easier, and computationally inexpensive, to evaluate the influence of the choice of $\mathcal{S}$ on the value of $\mathbb{T}$ at a given sampling location $\boldsymbol{z}$.

In the scalar (acoustic) case, similar remarks apply, with $\mathbb{T}(\boldsymbol{z} ; \mathcal{S}, T)$ recast in the form

$$
\mathbb{T}(\boldsymbol{z} ; \mathcal{S}, T)=\boldsymbol{\pi}(\boldsymbol{z}, T): \mathcal{B}(\mathcal{S}), \quad \text { with } \quad \boldsymbol{\pi}(\boldsymbol{z}, T)=[\boldsymbol{\nabla} u \star \boldsymbol{\nabla} \hat{u}](\boldsymbol{z}, T)
$$

Crack orientation. An important feature of the sought crack is its orientation, which is normally not known a priori. Let $\mathcal{R}$ denote an affine rotation that leaves the origin in $\overline{\boldsymbol{\xi}}$-space (i.e. $\boldsymbol{z}$ in the physical space) invariant and is otherwise characterized by the orthogonal matrix $\boldsymbol{R} \in \mathrm{SO}(3)$. Then, for a fixed shape of the trial infinitesimal crack $\mathcal{S}, \mathbb{T}(\boldsymbol{z} ; \mathcal{S}, T)$ depends on the chosen orientation of $\mathcal{S}$ via $\mathcal{A}$ (or $\mathcal{A}^{\sigma}$ ) through

$$
\mathcal{A}_{i j k \ell}(\mathcal{R}(\mathcal{S}))=R_{i I} R_{j J} R_{k K} R_{\ell L} \mathcal{A}_{I J K L}(\mathcal{S}),
$$

by virtue of the fact that evaluating (say) $\boldsymbol{\nabla} \boldsymbol{u}: \mathcal{A}(\mathcal{R}(\mathcal{S})): \boldsymbol{\nabla} \hat{\boldsymbol{u}}$ can be achieved by expressing $\boldsymbol{\nabla} \boldsymbol{u}, \boldsymbol{\nabla} \hat{\boldsymbol{u}}$ in the rotated frame $\left(\boldsymbol{E}_{1}, \boldsymbol{E}_{2}, \boldsymbol{E}_{3}\right)$ such that $\boldsymbol{E}_{i}=\boldsymbol{R}^{-1} \boldsymbol{e}_{i}$ and employing the original elastic moment tensor $\mathcal{A}(\mathcal{S})$. Then, in keeping with the previously-presented heuristic, it is natural to seek the orientation such that, for a given sampling point $\boldsymbol{z}$ and crack shape, $\mathbb{T}(\boldsymbol{z} ; \mathcal{S}, T)$ is lowest. Acordingly, define

$$
\begin{aligned}
& \mathbb{T}^{\mathrm{opt}}(\boldsymbol{z}, T)=\min _{\boldsymbol{R} \in \mathrm{SO}(3)} \mathbb{T}(\boldsymbol{z} ; \mathcal{R}(\mathcal{S}), T) \\
& \boldsymbol{R}^{\mathrm{opt}}(\boldsymbol{z}, T)=\underset{\boldsymbol{R} \in \mathrm{SO}(3)}{\arg \min } \mathbb{T}(\boldsymbol{z} ; \mathcal{R}(\mathcal{S}), T) \quad(\boldsymbol{z} \in \Omega) .
\end{aligned}
$$


If the trial crack $\mathcal{S}$ is penny-shaped, the minimization (48) reduces to a minimization w.r.t. the unit normal $\boldsymbol{n} \in \mathbb{S}$ (where $\mathbb{S}$ is the unit sphere), yielding an optimal unit normal $\boldsymbol{n}^{\text {opt }}(\boldsymbol{z}, T)$; the topological derivative will in this case be denoted $\mathbb{T}(\boldsymbol{z} ; \boldsymbol{n}, T)$ for emphasis. Furthermore, since $\mathcal{A}$ (or $\mathcal{A}^{\sigma}$ ) is in this case an even function of $\boldsymbol{n}$, the search space for $\boldsymbol{n}$ may be limited to one-half of $\mathbb{S}$.

In the acoustic case, involving a second-order polarization tensor, the minimization problem w.r.t. $\boldsymbol{n}$ is solvable exactly using an algebraic argument proposed e.g. in [10]. On noting $\boldsymbol{\nu}^{\min }(\boldsymbol{z}, T)$ the eigenvector corresponding to the smallest (real) eigenvalue of the symmetric tensor $\boldsymbol{\pi}(\boldsymbol{z}, T)$, the solution to (48) is

$$
\mathbb{T}^{\mathrm{opt}}(\boldsymbol{z}, T)=\mathbb{T}\left(\boldsymbol{z} ; \boldsymbol{\nu}^{\min }(\boldsymbol{z}, T), T\right), \quad \boldsymbol{n}^{\mathrm{opt}}(\boldsymbol{z}, T)=\boldsymbol{\nu}^{\min }(\boldsymbol{z}, T) \quad(\boldsymbol{z} \in \Omega) .
$$

The argument leading to (49) cannot be carried over to the elastic case. In the latter case, one needs to find a rotation $\hat{\mathcal{R}} \in \mathrm{SO}(6)$ (using Voigt-type $6 \times 6$ matrix representations of fourthorder tensors) such that the eigendirections of $\mathcal{A}$ can be rotated to match those of $\boldsymbol{\Pi}$. However, since $\mathcal{A}$ is defined in terms of a given crack surface $S$, one is only permitted to apply rotations $\mathcal{R} \in \mathrm{SO}(3)$ to $S$. As shown in [45], it is not always possible to find $\mathcal{R} \in \mathrm{SO}(3)$ inducing a given 6-dimensional rotation $\hat{\mathcal{R}} \in \mathrm{SO}(6)$, as $\mathcal{R}$ depends on three independent parameters (Euler's angles) while $\hat{\mathcal{R}}$ has 15 independent parameters. The minimization w.r.t. $\boldsymbol{n}$ is thus carried out numerically. As a side remark, the similar (but not identical) problem of finding directions of anisotropy that yield a pointwise optimal strain energy density is addressed in [48].

Normalized and thresholded topological derivative. To focus on sampling points $\boldsymbol{z} \in$ $\Omega$ where the topological derivative $\mathbb{T}^{\text {opt }}(\boldsymbol{z}, T)$ reaches sufficiently low (negative) values, and in view of the fact that the qualitative character of the TD lends significance to its relative, rather than absolute, values, a normalized and thresholded version $\hat{\mathbb{T}}_{\lambda}$ of $\mathbb{T}^{\text {opt }}$ is defined as

$$
\hat{\mathbb{T}}_{\lambda}(\boldsymbol{z}, T)=\left\{\begin{array}{ll}
\mathbb{T}^{\mathrm{opt}}(\boldsymbol{z}, T) /\left|\mathbb{T}^{\mathrm{min}}\right| & \text { if } \mathbb{T}^{\mathrm{opt}}(\boldsymbol{z}, T) \leq \lambda \mathbb{T}^{\mathrm{min}} \\
0 & \text { if } \mathbb{T}^{\mathrm{opt}}(\boldsymbol{z}, T)>\lambda \mathbb{T}^{\text {min }}
\end{array} \quad \text { with } \mathbb{T}^{\mathrm{min}}=\min _{\boldsymbol{z} \in \Omega} \mathbb{T}^{\mathrm{opt}}(\boldsymbol{z}, T),\right.
$$

(where $\lambda>0$ is a cut-off parameter and with the implicit assumption $\mathbb{T}^{\text {min }}<0$ ). Moreover, the support $S_{\lambda} \subset \Omega$ of $\hat{\mathbb{T}}_{\lambda}(\cdot, T)$, defined by

$$
S_{\lambda}=\left\{\boldsymbol{z} \in \Omega \mid \hat{\mathbb{T}}_{\lambda}(\boldsymbol{z}, T)<0\right\},
$$

can be considered as a reconstruction of the unknown crack (or set of cracks) suggested by the thresholded topological derivative.

Moreover, by analogy with the thresholded topological derivative (50) and assuming that $\mathcal{S}$ is the unit circular crack, let the subset $\mathbb{S}_{\alpha}^{\text {opt }}(\boldsymbol{z}, T)$ of the unit sphere $\mathbb{S}$ be defined for $\alpha \geq 0$ by

$$
\mathbb{S}_{\alpha}^{\text {opt }}(\boldsymbol{z}, T)=\left\{\boldsymbol{n} \in \mathbb{S}^{\mathrm{opt}}(\boldsymbol{z}, T) \mid \hat{\mathbb{T}}_{\lambda}(\boldsymbol{z}, \boldsymbol{n}, T) \leq \alpha\right\},
$$

where $\mathbb{S}^{\text {opt }}(\boldsymbol{z}, T):=\left\{\boldsymbol{n} \in \mathbb{S}, \boldsymbol{n}^{\text {opt }}(\boldsymbol{z}, T) \cdot \boldsymbol{n} \geq 0\right\}(\boldsymbol{z} \in \Omega)$ is the half-sphere oriented by $\boldsymbol{n}^{\text {opt }}(\boldsymbol{z}, T)$. The condition $\alpha \leq \lambda$ ensures that $\mathbb{S}_{\alpha}^{\text {opt }}(\boldsymbol{z}, T)$ is not empty when $\boldsymbol{z} \in S_{\lambda}$. Then, the average optimal normal $\boldsymbol{n}_{\lambda, \alpha}^{\mathrm{opt}}$ is defined at sampling points $\boldsymbol{z} \in S_{\lambda}$ by

$$
\boldsymbol{n}_{\lambda, \alpha}^{\mathrm{opt}}(\boldsymbol{z}, T)=A \int_{\mathbb{S}_{\alpha}^{\mathrm{opt}}(\boldsymbol{z}, T)} \boldsymbol{n} \mathrm{d} S_{\bar{\xi}} \quad\left(\boldsymbol{z} \in S_{\lambda}\right),
$$

(with the constant $A>0$ chosen so that $\boldsymbol{n}_{\lambda, \alpha}^{\text {opt }}$ has unit norm). If the cut-off parameters $\lambda$ and 
$\alpha$ entering definition (53) are chosen equal, the notation $\boldsymbol{n}_{\lambda}^{\text {opt }}$ will be used instead of $\boldsymbol{n}_{\lambda, \alpha}^{\text {opt }}$. In the acoustic case, the notation $\boldsymbol{n}_{\lambda}^{\text {opt }}(\boldsymbol{z}, T)$ will refer to the (exact) optimal normal $\boldsymbol{n}^{\text {opt }}(\boldsymbol{z}, T)$ at sampling points $\boldsymbol{z} \in S_{\lambda}$..

\section{NUMERICAL EXAMPLES}

In this section, numerical experiments on 3-D configurations are presented to evaluate the efficiency of the topological derivative indicator for crack detection, based on the least-squares cost function defined by (2) and (3), using synthetic transient dynamical measurements. In contrast with the somewhat involved analysis required to arrive at the correct formulation (26a) of the TD field, subsequent numerical implementations require only standard computational engineering methods. To emphasize the ease of application of the TD concept, all examples presented in this article were produced by means of straightforward displacement-based FEM formulations of the free and adjoint field, implemented using the FEM library CAST3M [24]. Since the behavior of the topological derivative method for cavity identification was studied through a rather exhaustive set of numerical experiments in [13], and expecting many findings of [13] to carry over to crack identification, the numerical experiments reported next focus on aspects pertaining to crack identification.

Discretization. Let $\Omega_{h}$ denote a FEM discretization of the reference domain $\Omega$. Moreover, synthetic data are generated using a discretized version $\Omega_{h}^{\star}$ of the "true" domain $\Omega^{\star}$ with the crack (or set thereof) to be identified. The meshes of $\Omega_{h}$ and $\Omega_{h}^{\star}$ do not coincide: to guard against the "inverse crime" [28], the meshes of $\Omega_{h}$ and $\Omega_{h}^{\star}$ are created independently (for convenience, the discretized observation surfaces $\Gamma_{\text {obs }, h}$ and $\Gamma_{\text {obs }, h}^{\star}$ were however arranged to geometrically coincide). The synthetic data $\boldsymbol{u}_{h}^{\text {obs }}$ is then defined as the restriction on $\Gamma_{\text {obs }, h}$ of the space-time discrete solution $\boldsymbol{u}_{h}^{\star}$ corresponding to the actual crack.

Once synthetic data are obtained, the evaluation of the field $\mathbb{T}$ as given by (44) requires the computation of the tensor field $\boldsymbol{\Pi}$ defined by (45). The discrete free and adjoint transient solutions entering $\Pi$ are both defined on the discretized reference domain $\Omega_{h}$. The present implementation is based on isoparametric piecewise-linear four-noded tetrahedral finite elements and the unconditionally-stable Newmark time-marching scheme with parameters $\beta=1 / 4, \gamma=$ $1 / 2$ [38]. The elastic moment tensor $\mathcal{A}^{\sigma}$ and the polarization tensor $\mathcal{B}$, appearing in (44) (resp. (47) are implemented using analytical formulae (33) (resp. (43), corresponding to the nucleation of an infinitesimal penny-shaped crack.

All numerical examples are defined in terms of non-dimensional space and time coordinates respectively defined by $\tilde{\boldsymbol{x}}=\boldsymbol{x} / \bar{\ell}$ and $\tilde{t}=t \bar{c} / \bar{\ell}$, with $\bar{\ell}$ and $\bar{c}$ denoting reference values for length and wave velocity. All lengths and durations will thereafter be implicitly understood as nondimensional, with the reference velocity $\bar{c}$ specified for each example and the tilde symbols on $\tilde{\boldsymbol{x}}, \tilde{t}$ or related quantities dropped. Moreover, for notational convenience, the Neumann portion $\Gamma_{\mathrm{N}}$ of $\Gamma$ will be split according to $\Gamma_{\mathrm{N}}=\Gamma_{\mathrm{L}} \cup \Gamma_{\mathrm{F}}$, where $\Gamma_{\mathrm{L}}$ is the support of a nonzero applied load density, the remaining part $\Gamma_{\mathrm{F}}$ being traction-free.

\subsection{Penny-shaped crack in a cubic domain}

The reference geometry $\Omega$ for this example, in a Cartesian coordinate system $\left(x_{1}, x_{2}, x_{3}\right)$, is the unit cube $0<x_{i}<1$, with $\Omega_{h}$ meshed using 27840 nodes. The crack $S^{\star}$ to be identified is a penny-shaped crack of radius $R=0.1$, centered at $\boldsymbol{x}^{\star}=(0.65,0.65,0.7)$ and with unit normal $\boldsymbol{n}^{\star}=-\sin \theta \boldsymbol{e}_{1}+\cos \theta \boldsymbol{e}_{3}$ (Fig. 1). The corresponding cracked domain $\Omega_{h}^{\star}$ is meshed using with 41062 nodes on average (slight variations occur depending on the chosen crack orientation $\theta$ ). The loading is applied on $\Gamma_{\mathrm{L}}=\left\{x_{3}=1\right\}$, while $\Gamma_{\mathrm{D}}=\emptyset, \Gamma_{\mathrm{F}}=\Gamma \backslash \Gamma_{\mathrm{F}}$ and $\Gamma_{\text {obs }}=\Gamma$ (observation available on the whole external boundary). The duration of the simulated experiment is set to 


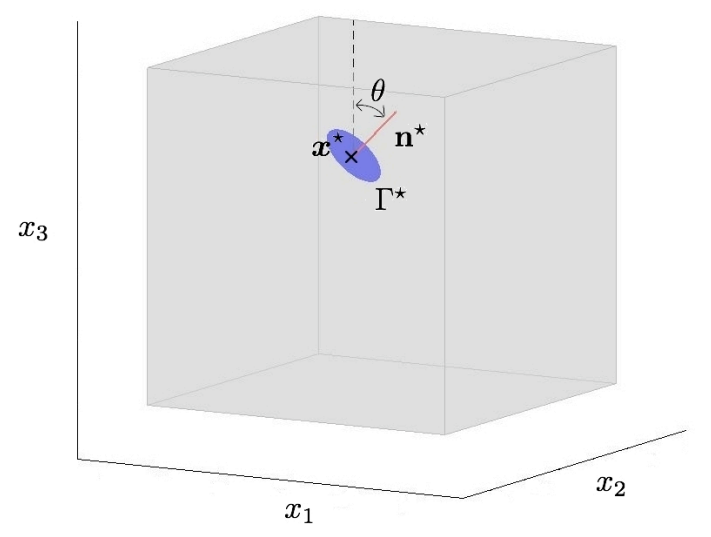

Figure 1: Cracked cubic domain

$T=2$. Both acoustic and elastic cases are considered for this example. Finally the indicator functions are represented on a subdomain of the reference domain $\Omega$, namely the cubic domain such that $\boldsymbol{x} \in[0.05 ; 0.95]^{3}$.

Acoustics. The applied load on $\Gamma_{\mathrm{L}}$ is given by $q\left[u^{\star}\right]=-H(t)$ (where $H$ denotes the Heaviside step function). The reference velocity is taken as the acoustic wave velocity of the medium, i.e. $\bar{c}=c$ (hence $T=2$ is the duration required for a plane wave emanating from a side to hit the opposite side and come back). The identification results are shown in Fig. 2 in terms of the normalized TD field $\hat{\mathbb{T}}_{0}$, the TD-based reconstruction $S_{\lambda}$ defined by (51) and the optimal normals $\boldsymbol{n}_{\lambda}^{\text {opt }}$ at sampling points, for three crack inclinations respectively defined by $\theta=0, \pi / 10, \pi / 4$. Qualitatively correct identification of the crack location, size and orientation is achieved.

Elasticity. A uniform compressional loading $\boldsymbol{t}\left[\boldsymbol{u}^{\star}\right]=-H(t) \boldsymbol{e}_{3}$ is imposed on $\Gamma_{\mathrm{L}}$. Consistently with this loading mode, the reference velocity $\bar{c}$ is chosen as the longitudinal wave velocity, i.e. $\bar{c}=c_{\mathrm{L}}=\kappa^{-1} \sqrt{\mu / \rho}$, with $\kappa$ given by (B.3). The identification results are shown in Fig. 3 in the same manner as for the acoustic case, with a qualitatively correct identification again achieved.

\subsection{Cylindrical shell containing a surface-breaking crack}

The reference geometry is now a cylindrical shell, defined in a cylindrical coordinate system $(r, \theta, z)$ by $\Omega=\left\{(r, \theta, z) \mid R_{i}<r<R_{e}, 0<z<H\right\}$, where the length, interior and exterior radii are respectively given by $H=4, R_{i}=0.9, R_{e}=1$, and with $\Omega_{h}$ meshed using 35747 nodes. A cracked configuration contains a crack $S^{\star}$ whose shape is a helicoidal strip, centered at $\boldsymbol{x}^{\star}$ and parametrized by its height $h$, depth $d$ and oriented angle $\alpha$ as indicated on Fig. 4, with $\alpha=\pi / 20, \pi / 5, \pi / 2$ used in the simulations. The crack breaks through the inner surface of the shell. The meshes $\Omega_{h}^{\star}$ generated for each of the three cracked geometries are refined in the crack vicinity (compared to the mesh of $\Omega_{h}$ ), and feature 43294, 49399 and 70426 nodes, respectively (a portion of the FE mesh for the case $\alpha=\pi / 2$ is depicted in Fig. 4). The observation surface is defined by $\Gamma_{\text {obs }}=\left\{r=R_{e}, 0<z<H\right\}$. Finally, the indicator functions are represented on the subdomain such that $r \in\left[R_{i}+10^{-3}, R_{e}-10^{-3}\right]$ and $z \in\left[10^{-3}, H-10^{-3}\right]$.

\subsubsection{Single experiment, noise-free data}

A first set of results is presented in this section for a configuration involving a single crack centered at $\boldsymbol{x}^{\star}=(0.945,0,2)$ and characterized by $h=0.5$ and $d=0.09$. Synthetic data are 


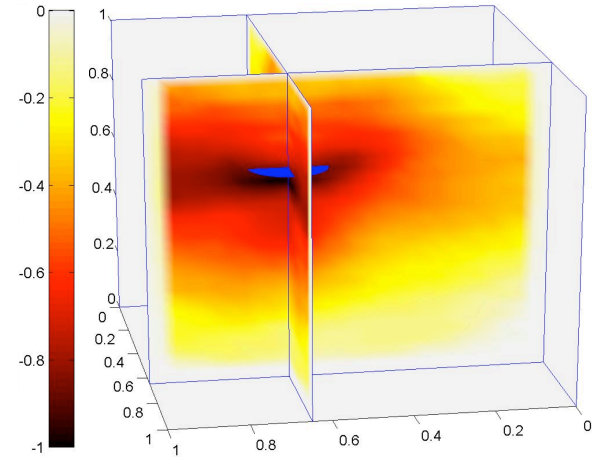

(a) $\theta=0$

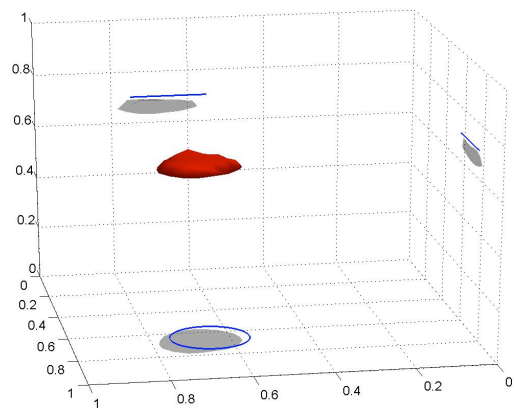

(d) $\theta=0, \lambda=0.9$

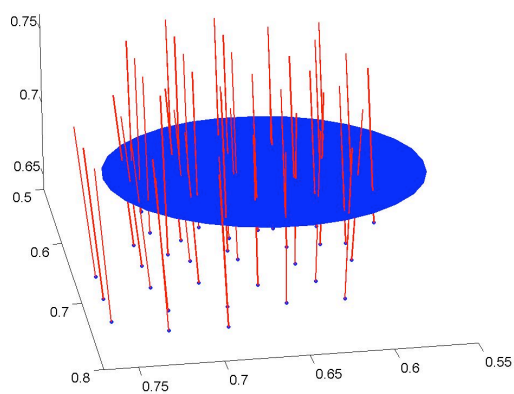

(g) $\theta=0, \lambda=0.9$

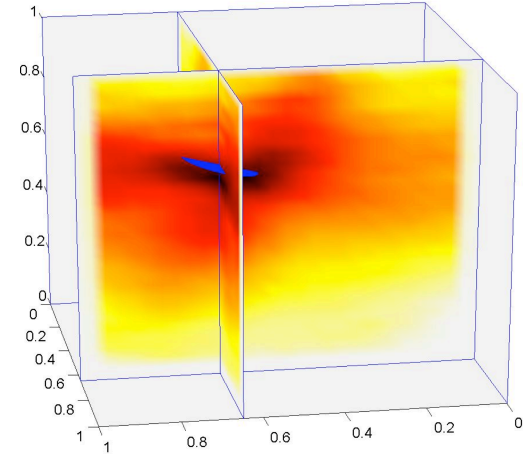

(b) $\theta=\pi / 10$

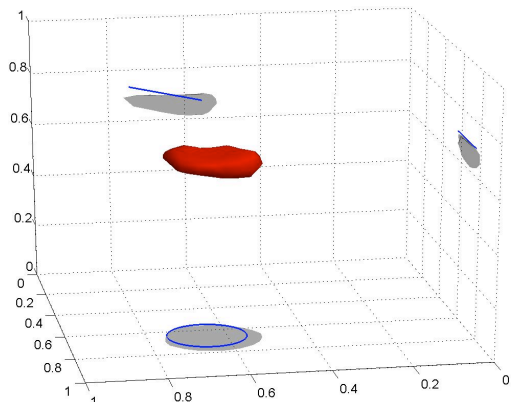

(e) $\theta=\pi / 10, \lambda=0.85$

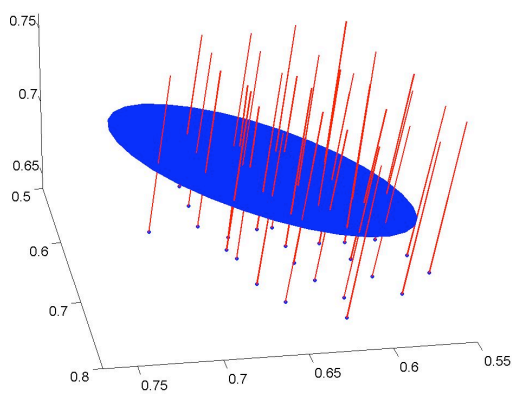

(h) $\theta=\pi / 10, \lambda=0.85$

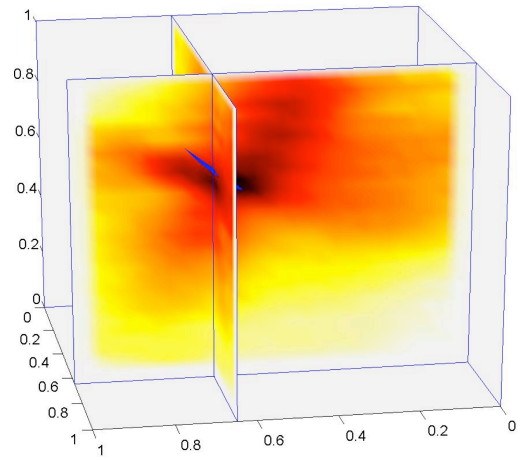

(c) $\theta=\pi / 4$

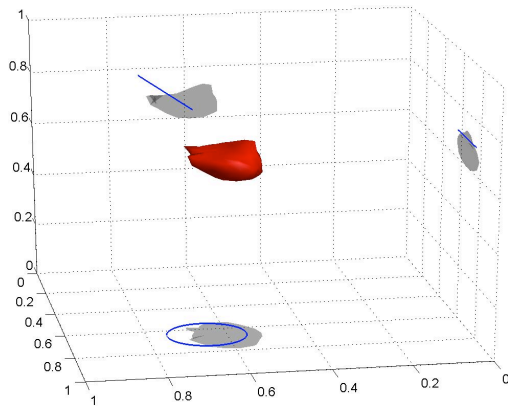

(f) $\theta=\pi / 4, \lambda=0.8$

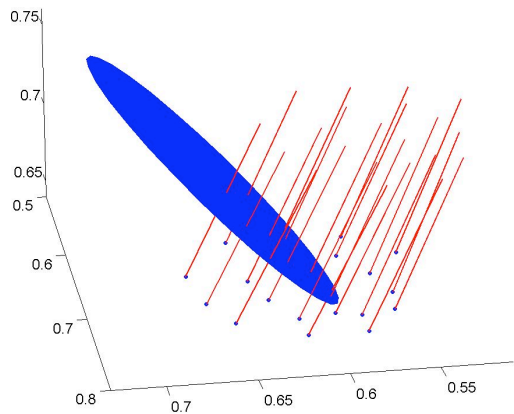

(i) $\theta=\pi / 4, \lambda=0.8$

(g)-(i): Optimal normals $\boldsymbol{n}_{\lambda}^{\text {opt }}$ at sampling points

Figure 2: Identification of a penny-shaped rigid screen in an acoustic unit cube. (a)-(c): normalized TD field $\hat{\mathbb{T}}_{0} ;(d)-(f)$ : reconstructed domain $S_{\lambda}$; (g)-(i): optimal normals $\boldsymbol{n}_{\lambda}^{\text {opt }}$ at sampling points

produced using a unique experiment, for which a loading combining traction and shear, given by

$$
\boldsymbol{t}\left[\boldsymbol{u}^{\star}\right]=\left(\sin \theta \boldsymbol{e}_{1}-\cos \theta \boldsymbol{e}_{2}+\boldsymbol{e}_{3}\right) g(t, 1,0.3) \quad \text { with } g(t ; m, s):=\exp \left(-\frac{(t-m)^{2}}{2 s^{2}}\right) H(t) .
$$

is applied on $\Gamma_{\mathrm{L}}$, while $\Gamma_{\mathrm{D}}=\emptyset$. In the expectation that the loading mode will trigger both compressional and shear waves, the reference velocity is chosen as that of shear waves, i.e. $\bar{c}=c_{\mathrm{T}}=\sqrt{\mu / \rho}$. The experiment duration is set to $T=8$. The identification results are shown for the three crack geometries in Fig. 5 , in terms of the normalized TD field $\hat{\mathbb{T}}_{0}$, the TD-based reconstruction $S_{\lambda}$ and the optimal normals $\boldsymbol{n}_{\lambda}^{\text {opt }}$ at sampling points. Satisfactory identification of the crack location and orientation is again achieved. 


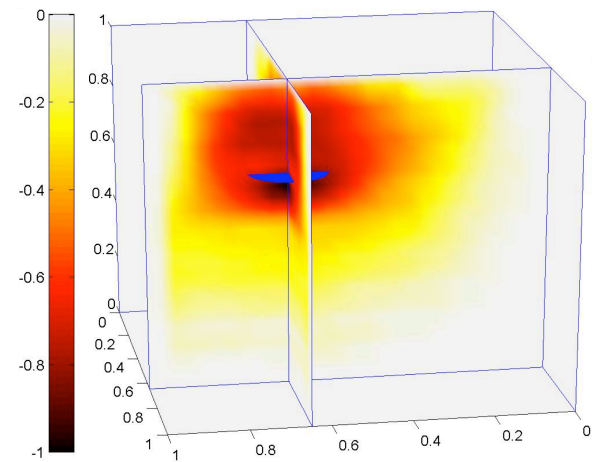

(a) $\theta=0$

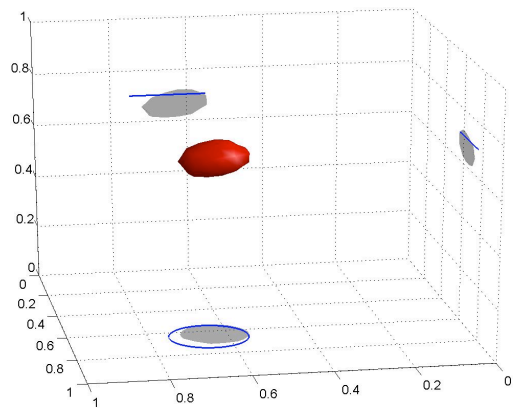

(d) $\theta=0, \lambda=0.8$

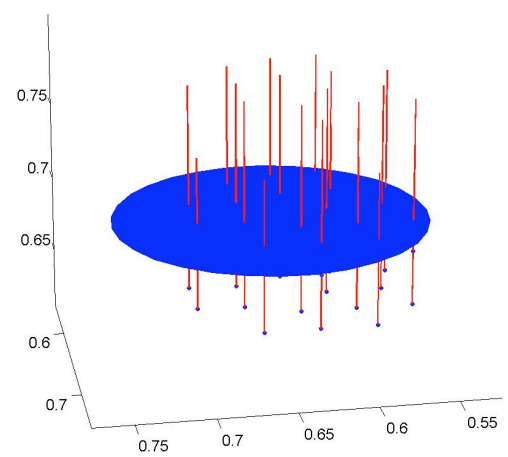

(g) $\theta=0, \lambda=0.8$

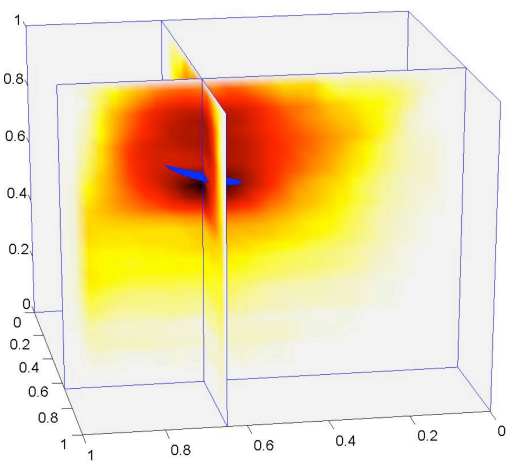

(b) $\theta=\pi / 10$

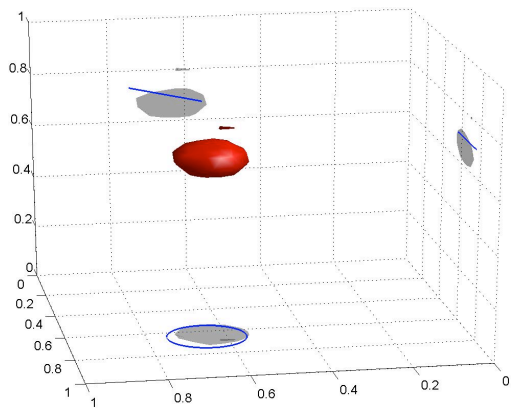

(e) $\theta=\pi / 10, \lambda=0.8$

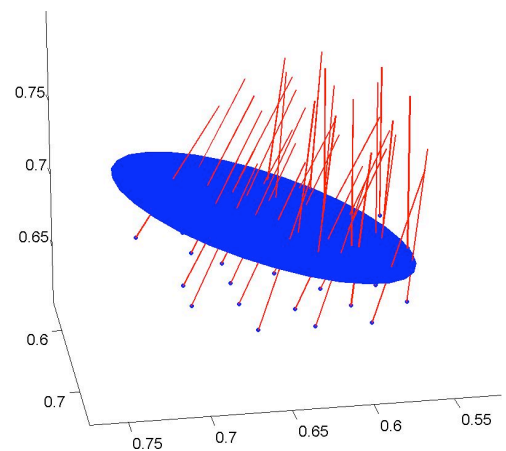

(h) $\theta=\pi / 10, \lambda=0.8$

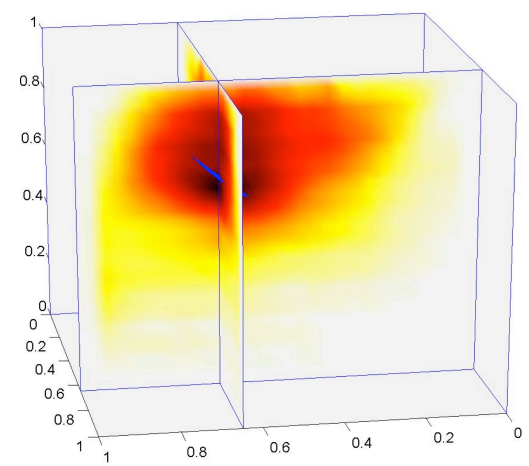

(c) $\theta=\pi / 4$

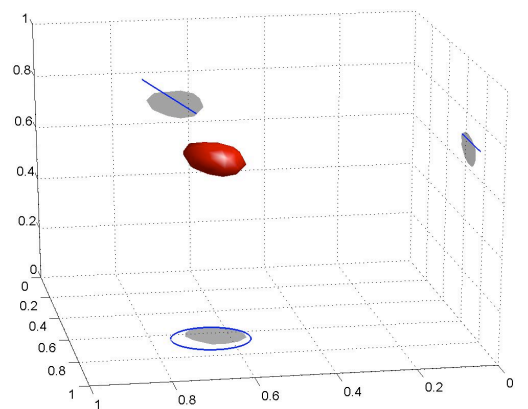

(f) $\theta=\pi / 4, \lambda=0.85$

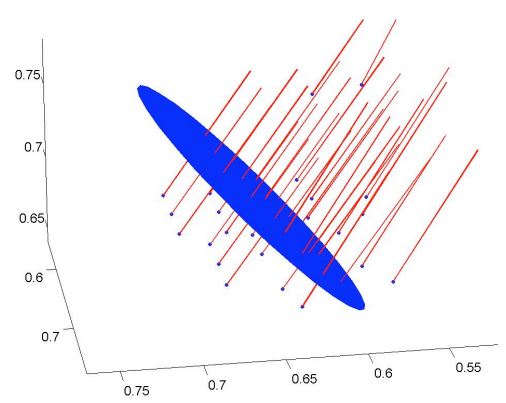

(i) $\theta=\pi / 4, \lambda=0.85$

Figure 3: Identification of a penny-shaped crack in an elastic unit cube. (a)-(c): normalized TD field $\hat{\mathbb{T}}_{0} ;(d)-(f)$ : reconstructed domain $S_{\lambda} ;(g)-(i)$ : optimal normals $\boldsymbol{n}_{\lambda}^{\text {opt }}$ at sampling points

\subsubsection{Single experiment, noisy data}

The influence of data noise is evaluated by considering noisy synthetic data of the form

$$
\boldsymbol{u}_{\mathrm{obs}}(\boldsymbol{\xi}, t)=\boldsymbol{u}_{h}^{\star}(\boldsymbol{\xi}, t)+\sigma \chi(\boldsymbol{\xi}, t)\left\|\boldsymbol{u}_{h}^{\star} \cdot \boldsymbol{e}_{k}\right\|_{L^{\infty}\left(S_{\mathrm{obs}} \times[0, T]\right)} \boldsymbol{e}_{k},
$$

where $\chi$ is a Gaussian random variable with zero mean and unit standard deviation and $\sigma$ denotes the fixed noise level. Results obtained on the configuration of Fig. 4 with $\alpha=\pi / 5$ for noise levels $\sigma=0.01,0.05,0.1$ are presented in Fig. 6 . Note that one has for the considered configuration:

$$
\max _{k=1, \ldots, 3} \frac{\|\left(\boldsymbol{u}-\boldsymbol{u}_{h}^{\star} \cdot \boldsymbol{e}_{k} \|_{L^{\infty}\left(S_{\mathrm{obs}} \times[0, T]\right)}\right.}{\left\|\boldsymbol{u}_{h}^{\star} \cdot \boldsymbol{e}_{k}\right\|_{L^{\infty}\left(S_{\mathrm{obs}} \times[0, T]\right)}}=6.05 \cdot 10^{-2}
$$



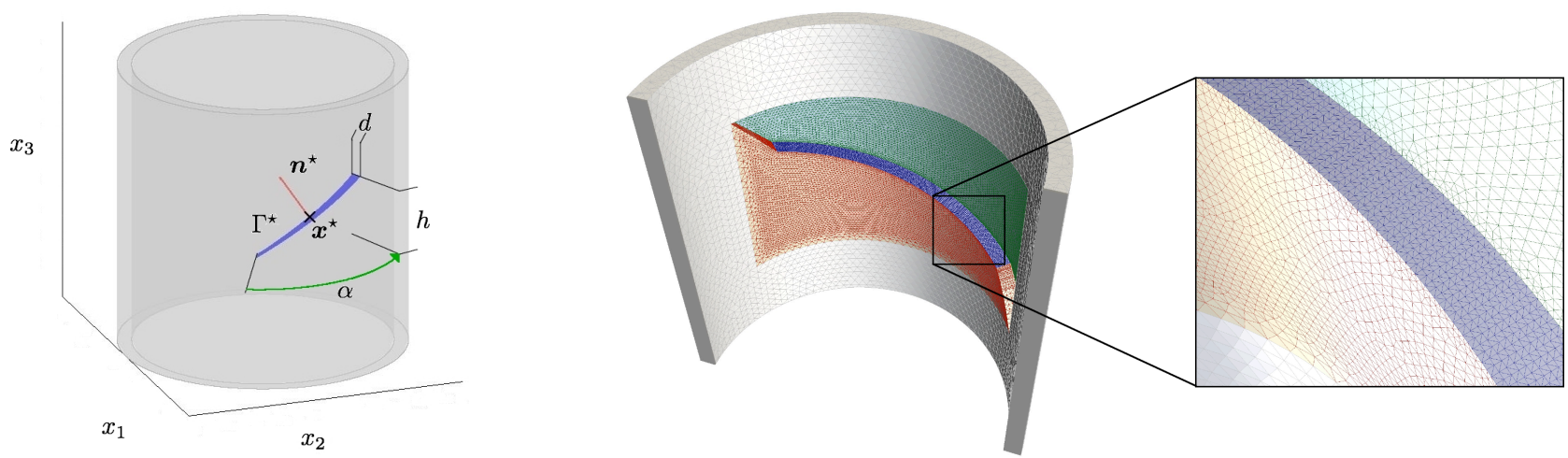

Figure 4: Cracked cylindrical shell: geometry and notation (left), portion of the FE mesh for the case $\alpha=\pi / 2$ (right)

which shows that the relative noise corrupting the difference $\boldsymbol{u}-\boldsymbol{u}_{h}^{\star}$, which constitutes the useful experimental scattered field information but cannot be measured directly, is roughly 15 times larger than the relative noise affecting the total displacement $\boldsymbol{u}_{h}^{\star}$.

\subsubsection{Cumulated experiments}

In this section, four different (simulated) experiments, differing by the choice of the loading zone $\Gamma_{\mathrm{L}}$, are combined to image a single crack characterized by $\boldsymbol{x}^{\star}=(0.945,0,2), h=0.5$, $d=0.09$ and $\alpha=\pi / 5$. The loading zone $\Gamma_{\mathrm{L}}^{(k)}$ for experiment number $k(1 \leq k \leq 4)$ is a small patch of surface area $10^{-3}\left|\Gamma_{\text {obs }}\right|$ (where $\left|\Gamma_{\text {obs }}\right|=2 \pi R_{e} H$ is the area of the whole observation surface), centered at the point $P^{(k)}$ whose cylindrical coordinates are given in Table 1 . To avoid spurious effects for the topological derivative computed in the patch area, an additional neighborhood of the point $P_{k}$ is excluded for the representation of the indicator function $\hat{\mathbb{T}}_{0}$. The experiment duration is set to $T=4$, i.e. half the duration used in the simgle-experiment case. The topological derivative is computed by means of $\boldsymbol{\Pi}(\boldsymbol{z}, T)$ given by (46) with $K=4$. In experiment $k$, a shear loading given by $\boldsymbol{t}\left[\boldsymbol{u}^{\star}\right](\boldsymbol{\xi}, t)=\left(\sin \theta \boldsymbol{e}_{1}-\cos \theta \boldsymbol{e}_{2}+\boldsymbol{e}_{3}\right) g(t, 1,0.3)$ is applied on the patch $\Gamma_{\mathrm{L}}^{(k)}$ (with the time modulation $g$ again defined by (54)), while the reference velocity is again chosen as $\bar{c}=c_{\mathrm{T}}=\sqrt{\mu / \rho}$. Results are shown on Fig. 7. The crack identification is seen to improve with the number $K$ of experiments, and is noticeably better in the case $K=4$ than for the longer $(T=8)$ single experiment reported for the same sought crack geometry (but different loading conditions) in Fig. 5b. Pronounced negative values of the topological derivative also occur away from the crack location for this example, however; these are in fact located in the vicinity of one of the loading patches. This may be caused by a lack of smoothness of the free field near the loading zone, making the topological asymptotic invalid in this region (see Remark 6).

\begin{tabular}{ccccc}
\hline Patch center & $P^{(1)}$ & $P^{(2)}$ & $P^{(3)}$ & $P^{(4)}$ \\
\hline$r$ & 1 & 1 & 1 & 1 \\
$\theta$ & $\pi / 3$ & $-\pi / 3$ & $2 \pi / 3$ & $-2 \pi / 3$ \\
$z$ & 3.5 & 0.5 & 1.25 & 2.75
\end{tabular}

Table 1: Multiple excitation: loading patch center $P^{(k)}$ for experiment $k$ 


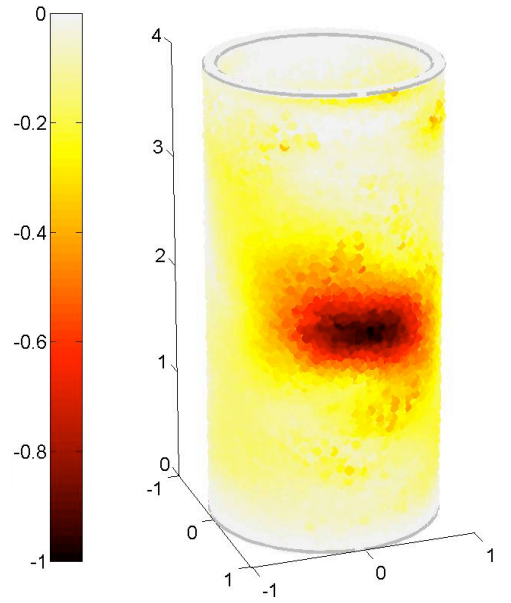

(a) $\alpha=\pi / 20$

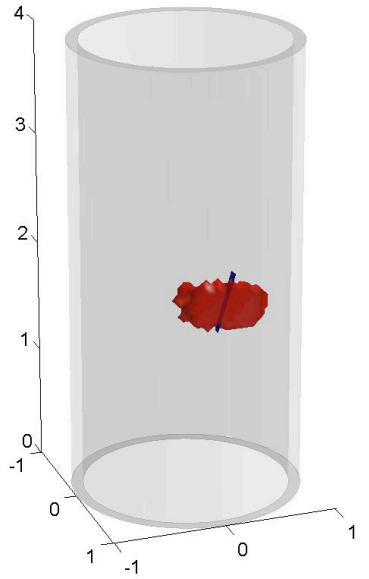

(d) $\alpha=\pi / 20$

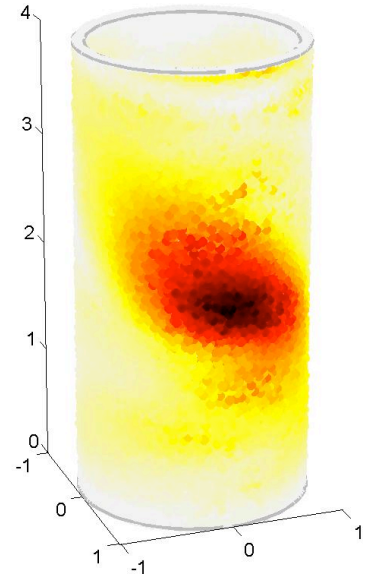

(b) $\alpha=\pi / 5$

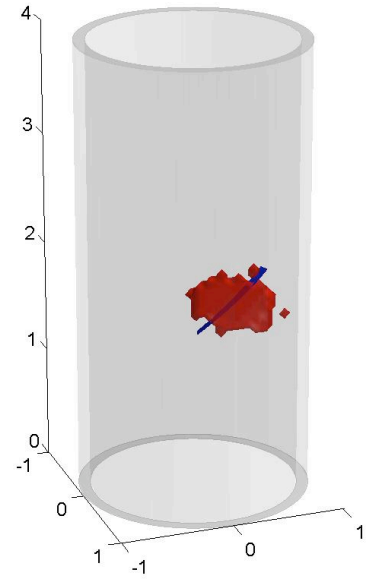

(e) $\alpha=\pi / 5$

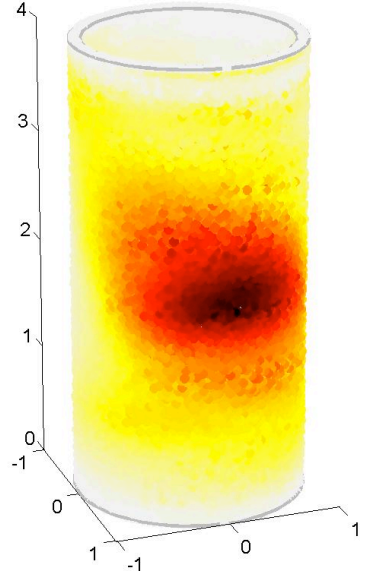

(c) $\alpha=\pi / 2$

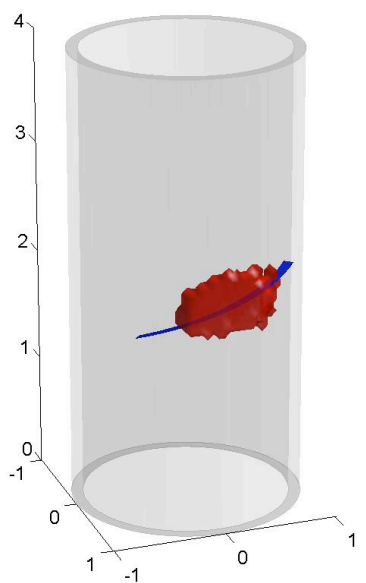

(f) $\alpha=\pi / 2$

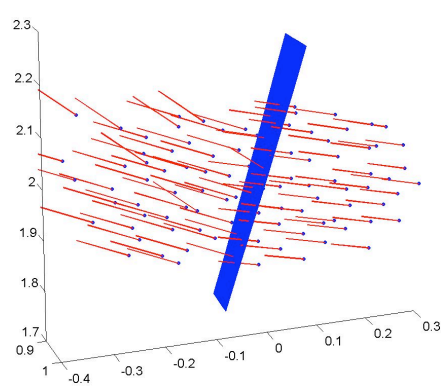

(g) $\alpha=\pi / 20$

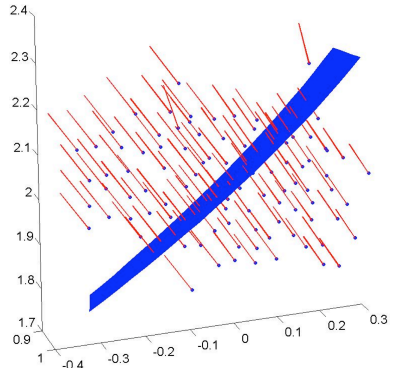

(h) $\alpha=\pi / 5$

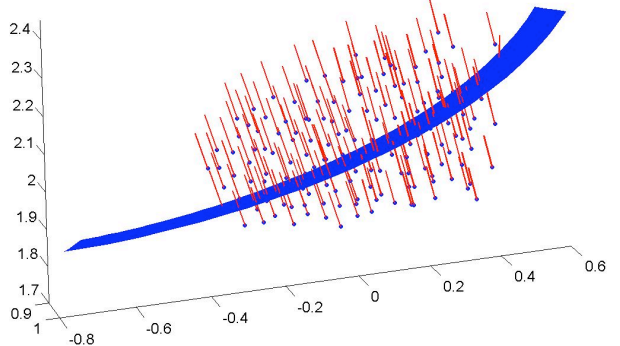

(i) $\alpha=\pi / 2$

Figure 5: Cylindrical shell (elastic), single experiment: identification of a single crack. (a)(c): normalized $T D$ field $\hat{\mathbb{T}}_{0} ;(d)-(f)$ : reconstructed domain $S_{0.8} ;(g)-(i)$ : optimal normals $\boldsymbol{n}_{0.8,0}^{\text {opt }}$ at sampling points

\subsubsection{Double crack identification}

A single experiment is now used to image a configuration containing two helicoidal crack centered at $\boldsymbol{x}_{1}^{\star}=(0.945, \pi / 3,0.75)$ and $\boldsymbol{x}_{2}^{\star}=(0.945, \pi / 3,3)$, respectively characterized by the angles $\alpha_{1}=\pi / 4$ and $\alpha_{2}=-\pi / 10$, and length parameters $h=0.5, d=0.09$. The discretized domains $\Omega_{h}$ and $\Omega_{h}^{\star}$ feature 36468 and 64416 nodes, respectively. The loading and experiment duration are the same as in Sec. 5.2.1. 


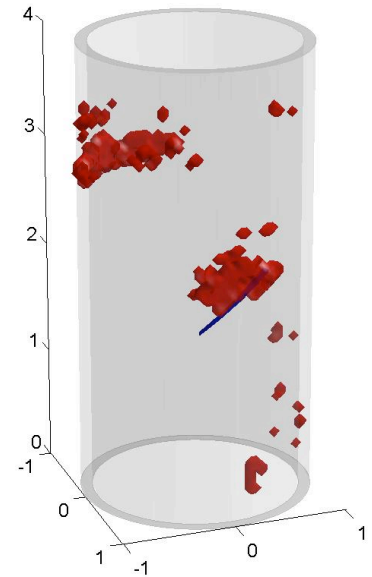

(a) $\sigma=0.01$

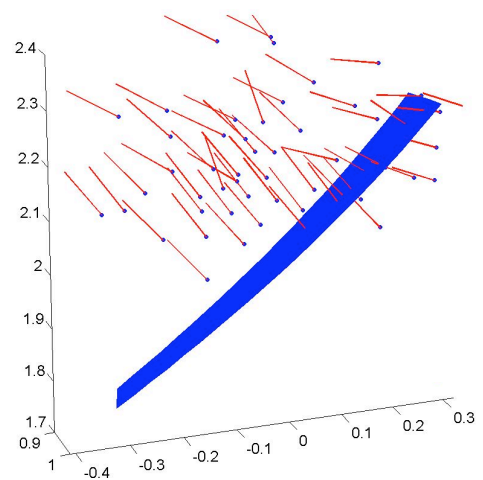

(d) $\sigma=0.01$

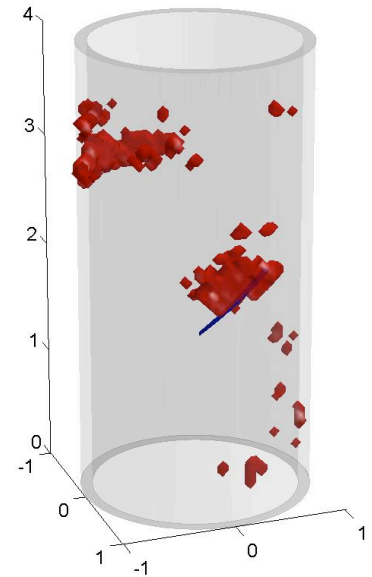

(b) $\sigma=0.05$

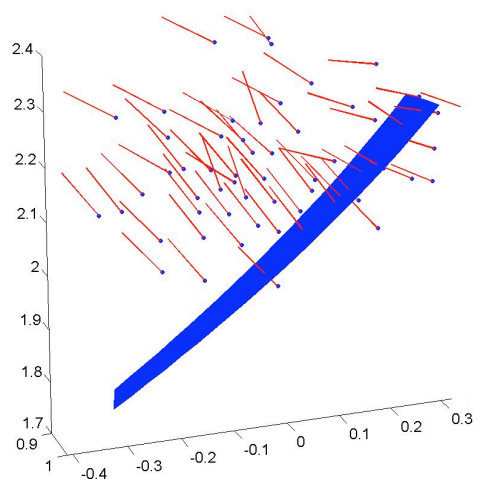

(e) $\sigma=0.05$

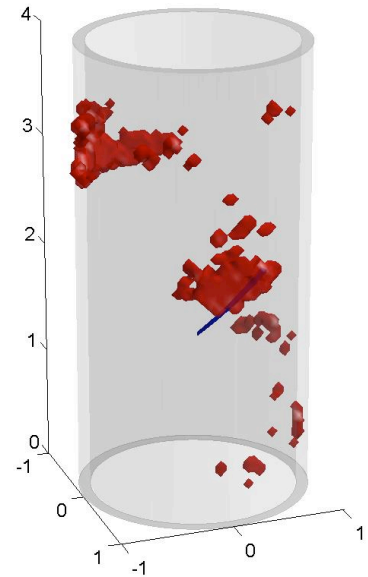

(c) $\sigma=0.1$

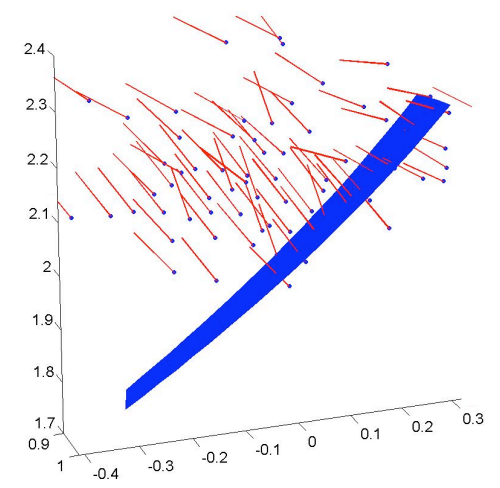

(f) $\sigma=0.1$

Figure 6: Cylindrical shell (elastic), single experiment: identification of a single crack, noisy data. (a)-(c): reconstructed domain $S_{0.7} ;(d)-(f)$ : optimal normals $\boldsymbol{n}_{0.7,0}^{o p t}$ at sampling points

\subsubsection{Elastic moment tensor defined in terms of an elliptical crack}

In all previous examples, the topological derivative was defined in terms of the elastic moment tensor (33) corresponding to the nucleation of a circular planar crack (or its acoustic counterpart (43). To evaluate the influence of the choice of the crack shape $\mathcal{S}$, the single-crack, singleexperiment example of Sec. 5.2.1 is now revisited by using the elastic moment tensor (32) for an elliptical crack in (44). The unknown crack $S^{\star}$ is characterized by $\alpha=\pi / 5$. The elastic moment tensor (32) (and the topological derivative (44) is computed in the $\left(\boldsymbol{\tau}_{1}^{\star}, \boldsymbol{\tau}_{2}^{\star}, \boldsymbol{n}^{\star}\right)$ orthonormal frame defined such that $\boldsymbol{n}^{\star}:=(0,-h / L, \alpha R / L)$ (in cartesian coordinates) is the correct normal to $S^{\star}$ and the tangential vectors $\boldsymbol{\tau}_{1}^{\star}, \boldsymbol{\tau}_{2}^{\star}$ are given by $\boldsymbol{\tau}_{1}^{\star}=(1,0,0), \boldsymbol{\tau}_{2}^{\star}=(0, \alpha R / L, h / L)$ (with $R=\left(R_{i}+R_{e}\right) / 2$ and $\left.L=\left(h^{2}+\alpha^{2} R^{2}\right)^{1 / 2}\right)$.

Figure 9 shows that the reconstructions $S_{0.8}$ obtained using $\mathcal{A}^{\sigma}(\mathcal{S})$ for (i) $\ell_{1}=\ell_{2}=1$ (pennyshaped trial crack), (ii) $\ell_{1}=4, \ell_{2}=1$ (elliptical crack elongated along the true crack length), or (iii) $\ell_{1}=1, \ell_{2}=4$ (elliptical crack elongated along the true crack depth) are very similar. In other words, the choice of trial crack shape $\mathcal{S}$ does not appear to affect much the estimation of the actual crack $S^{\star}$. Note that none of the trial shapes is close to the actual shape.

\subsection{Discussion}

The results corroborate the heuristic of the topological derivative method. The maps of topological derivative (Fig. 2a-c for the acoustic case, and Figs. 3a-c, 5a-c, 7a-c, 8b for the elastic case) reveal global negative minima in the areas of the cracks sought. The use of a truncation 


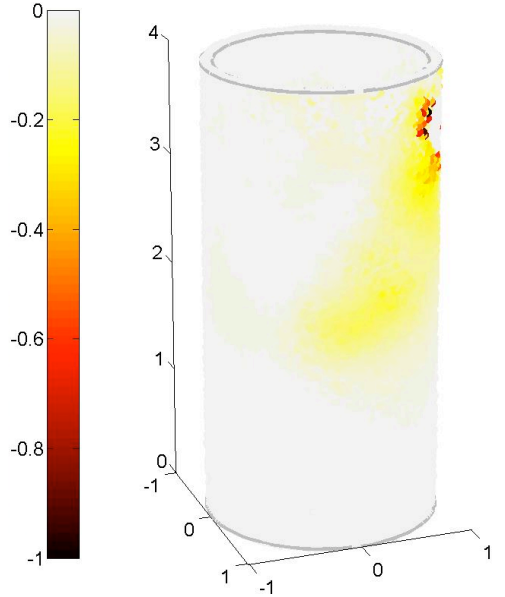

(a) Exp. $\{1\}$

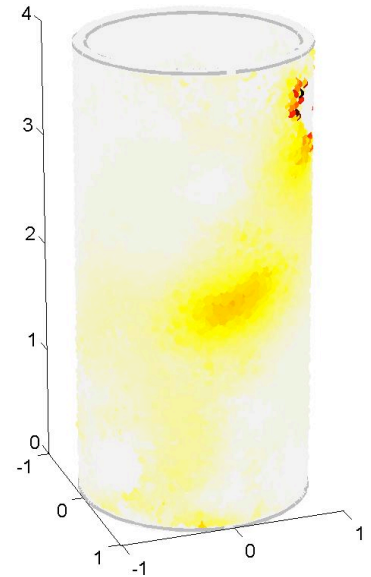

(b) Exp. $\{1,2\}$

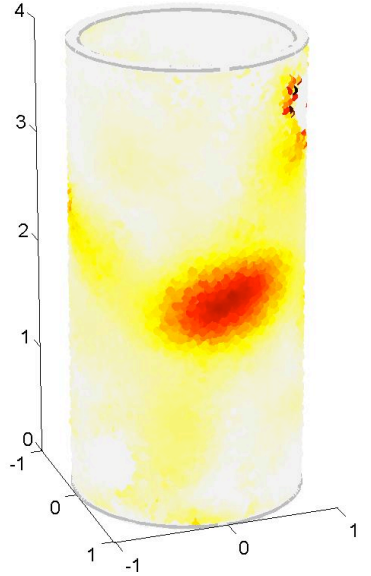

(c) Exp. $\{1,2,3,4\}$

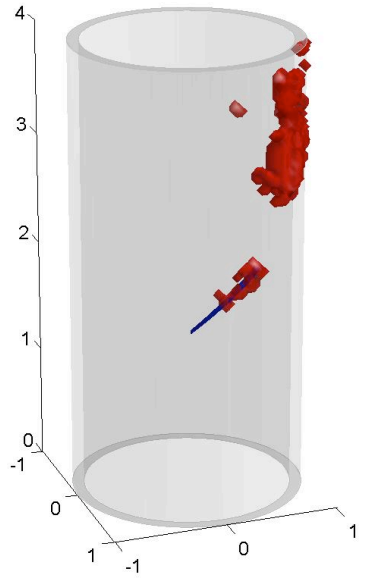

(d) Exp. $\{1\}, \lambda=0.2$

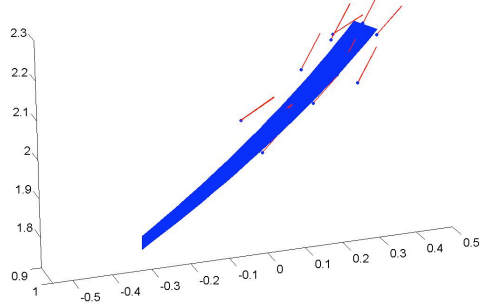

(g) Exp. $\{1\}, \lambda=0.2$

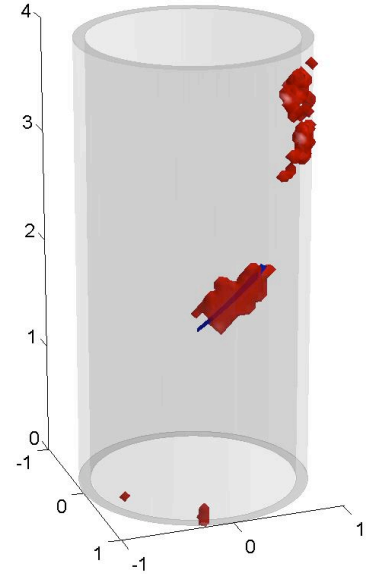

(e) Exp. $\{1,2\}, \lambda=0.3$

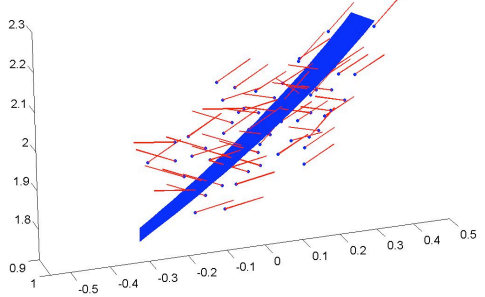

(h) Exp. $\{1,2\}, \lambda=0.3$

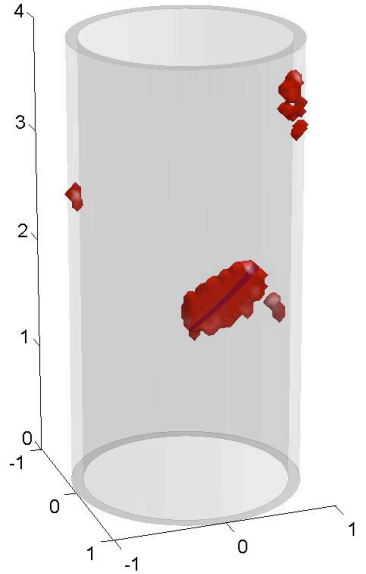

(f) Exp. $\{1,2,3,4\}, \lambda=0.55$

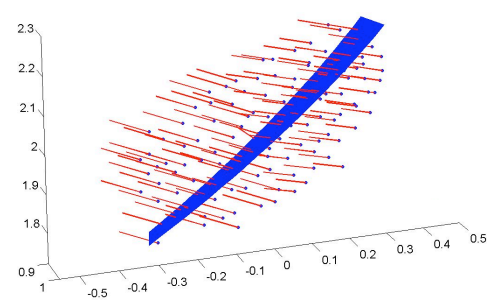

(i) Exp. $\{1,2,3,4\}, \lambda=0.55$

Figure 7: Cylindrical shell, cumulated experiments: identification of a single crack. (a)-(c): normalized TD field $\hat{\mathbb{T}}_{0} ;(d)-(f)$ : reconstructed domain $S_{\lambda}$; (g)-(i): optimal normals $\boldsymbol{n}_{\lambda, 0}^{\text {opt }}$ at sampling points

parameter $\lambda$ to reveal possible cracks geometries gives satisfactorily results in that the domains $S_{\lambda}$ are correctly located (Figs. 2d-f, 3d-f, 5d-f, 6a-c, 7d-f, 8c), while the normal vectors $\boldsymbol{n}_{\lambda, \alpha}^{\text {opt }}$ are reasonably consistent with the actual crack orientations (Figs. 2g-i, 3g-i, 5g-i, 6d-f, 7g-i, 8d,e). Moreover, while the geometry reconstructions $S_{\lambda}$ are bulky, i.e. do not reflect well the flat crack geometries, the orientation estimation through $\boldsymbol{n}_{\lambda, \alpha}^{\text {opt }}$ help resolve the ambiguity. Finally, figure 8 illustrates the global character of the topological derivative method as it enables a simultaneous identification of multiple cracks without prior knowledge of their number. The method proposed leads to a qualitatively correct identification of cracks even in situations where a single transient experiment is used. Additively combining several experiments improves the quality of the reconstruction (Fig. 7). 


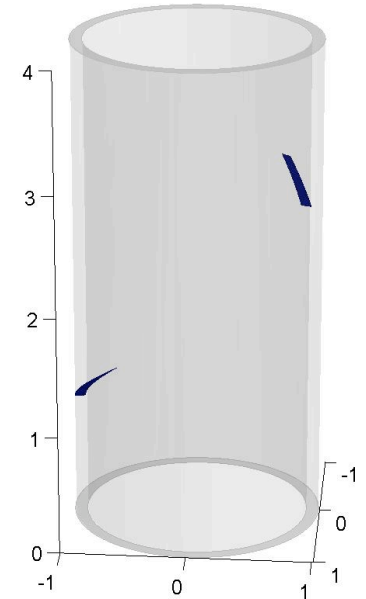

(a) Cracked configuration

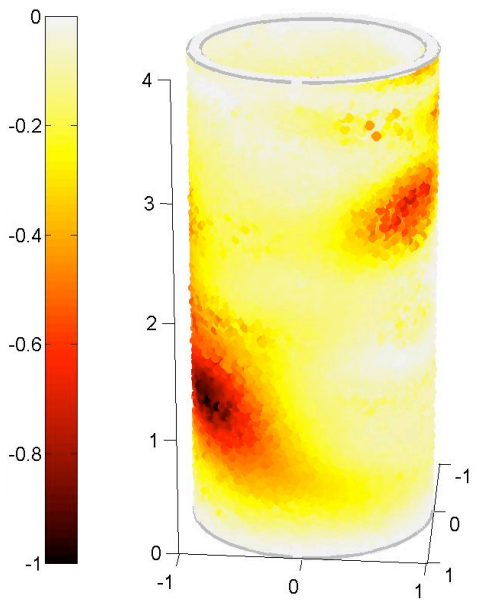

(b) Normalized TD field $\hat{\mathbb{T}}_{0}$

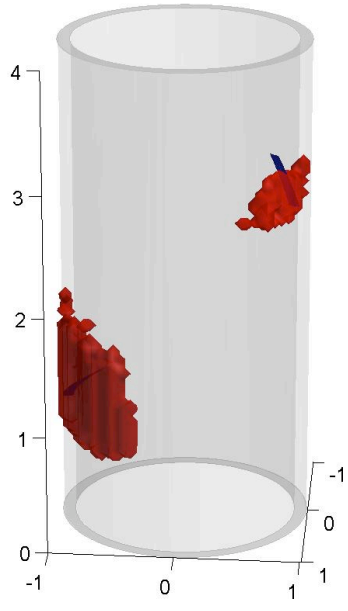

(c) Reconstructed domain $S_{0.5}$

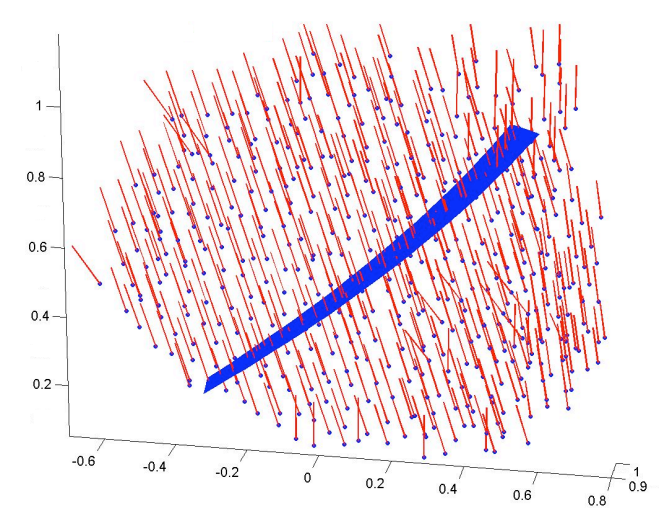

(d) Optimal normals $\boldsymbol{n}_{0.5}^{\mathrm{opt}}$ : crack 1

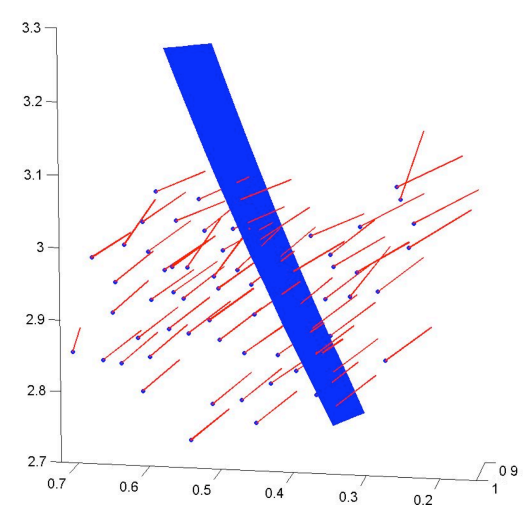

(e) Optimal normals $\boldsymbol{n}_{0.5}^{\mathrm{opt}}$ : crack 2

Figure 8: Cylindrical shell, single experiment: identification of a double crack

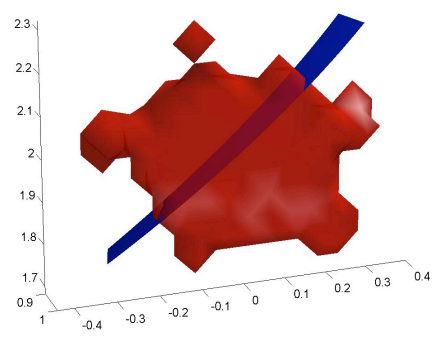

(a) $\ell_{1} / \ell_{2}=1$

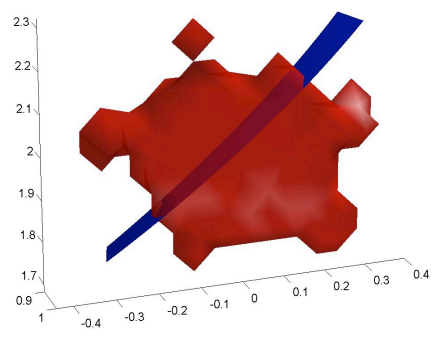

(b) Major axis: $\tau_{1}^{\star}, \ell_{1} / \ell_{2}=4$

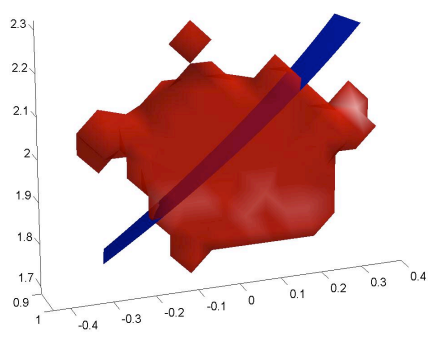

(c) Major axis: $\tau_{2}^{\star}, \ell_{1} / \ell_{2}=$ $1 / 4$

Figure 9: Infinitesimal elliptical crack: reconstruction $S_{0.8}$ for several choices of the elastic moment tensor

\section{CONCLUSION}

A non-iterative global qualitative crack identification approach exploiting transient elastodynamic data and based on the concept of topological derivative has been formulated. Its feasibility and usefulness have been demonstrated on numerical experiments. Some technical issues, such as practical procedures for computing relevant elastic moment tensors, have been addressed along the way. The proposed adjoint-based formulation requires solving two transient elastodynamic problems defined on the crack-free configuration, and therefore entails a computational cost equivalent to that of one single evaluation of the cost functional and its gradient in a 
traditional minimization-based inversion. While less accurate than a complete (topological and shape optimization-based) inversion, it is therefore also much faster. The indicator function based on the topological derivative field is of global nature in that it tests all potential crack locations in a chosen region of interest without requiring any initial guess. The implemented version of the approach was based on the elastic moment tensor for penny-shaped cracks, but was nevertheless able to locate actual cracks whose shape substantially deviate from the trial shape used for formulating the small-crack asymptotic. Moreover, the normal $\boldsymbol{n}_{\lambda, \alpha}^{\mathrm{opt}}$ obtained from the pointwise optimization of $\mathbb{T}(\boldsymbol{z} ; \boldsymbol{n}, T)$ was found to be reasonably consistent with the orientation of the sought $\operatorname{crack}(\mathrm{s})$, which constitutes another useful feature of the proposed approach. Finally, an implementation within a classical FEM platform highlighted the simplicity and efficiency of the method once the correct expression of the topological derivative is available.

Important theoretical and practical work still remains to be done. From a theoretical standpoint, the formulation presented herein requires deeper mathematical analysis for a rigorous justification of expansion (6) and possible limitations of its validity in terms of e.g. frequency content of the excitation. Besides, the method being now demonstrated as feasible from a computational standpoint, it is now clearly necessary to assess it against experimental data. Both avenues will be pursued in the near future.

\section{Appendix A ELASTIC MOMENT TENSORS}

\section{Appendix A.1 Major symmetry of elastic moment tensor (proof of Lemma 4)}

The proof rests upon exploiting a governing weak formulation for the third-order tensor function $\mathcal{V}(\overline{\boldsymbol{\xi}})$, which is such that for any given second-order symmetric constant tensor $\boldsymbol{\sigma} \in \mathbb{R}_{\text {sym }}^{3,3}$ the vector field $\boldsymbol{\sigma}: \mathcal{V}(\overline{\boldsymbol{\xi}})$ solves the elastostatic exterior problem for the normalized crack $\mathcal{S}$ whose faces are subjected to applied tractions $\boldsymbol{t}^{ \pm}=-\boldsymbol{\sigma} \cdot \boldsymbol{n}^{ \pm}$. As a result, $\boldsymbol{\sigma}: \mathcal{V}(\overline{\boldsymbol{\xi}})$ obeys for any $\boldsymbol{\sigma}$ the weak formulation

$$
\int_{\mathbb{R}^{3} \backslash \mathcal{S}}[\boldsymbol{\sigma}: \nabla \mathcal{V}]: \mathcal{C}: \nabla \boldsymbol{w} \mathrm{d} V_{\bar{\xi}}=\int_{\mathcal{S}}(\Delta \boldsymbol{w} \otimes \boldsymbol{n}): \boldsymbol{\sigma} \mathrm{d} S_{\bar{\xi}} \quad \forall \boldsymbol{w} \in H^{1}\left(\mathbb{R}^{3} \backslash \mathcal{S}\right),
$$

where $\Delta \boldsymbol{w}$ denotes the jump of $\boldsymbol{w}$ across $\mathcal{S}$ (note that the decay properties at infinity of $\mathcal{V}$, which directly follow from an integral representation formula, are such that $\mathcal{V} \in H^{1}\left(\mathbb{R}^{3} \backslash \mathcal{S}\right)$ ). Next, setting $\boldsymbol{w}=\boldsymbol{\sigma}^{\prime}: \mathcal{V}$ in the above identity, one obtains

$$
\int_{\mathbb{R}^{3} \backslash \mathcal{S}}[\boldsymbol{\sigma}: \nabla \mathcal{V}]: \mathcal{C}:\left[\boldsymbol{\sigma}^{\prime}: \nabla \mathcal{V}\right] \mathrm{d} V_{\bar{\xi}}=\boldsymbol{\sigma}^{\prime}:\left\{\int_{\mathcal{S}} \Delta \mathcal{V} \otimes \boldsymbol{n} \mathrm{d} S_{\bar{\xi}}\right\}: \boldsymbol{\sigma}=\boldsymbol{\sigma}^{\prime}: \mathcal{A}^{\sigma}: \boldsymbol{\sigma},
$$

with the last equality in (A.1) directly stemming from the definition (22) of $\mathcal{A}^{\sigma}$. Finally, noting that

$$
[\sigma: \nabla \mathcal{V}]: \mathcal{C}:\left[\sigma^{\prime}: \nabla \mathcal{V}\right]=\left[\sigma^{\prime}: \nabla \mathcal{V}\right]: \mathcal{C}:[\sigma: \nabla \mathcal{V}]
$$

by virtue of the well-known major and minor symmetries of $\mathcal{C},(\mathrm{A} .1)$ implies the claimed major symmetry of $\mathcal{A}^{\sigma}$ through

$$
\boldsymbol{\sigma}^{\prime}: \mathcal{A}^{\sigma}: \boldsymbol{\sigma}=\int_{\mathbb{R}^{3} \backslash \mathcal{S}}[\boldsymbol{\sigma}: \nabla \mathcal{V}]: \mathcal{C}:\left[\boldsymbol{\sigma}^{\prime}: \nabla \mathcal{V}\right] \mathrm{d} V_{\bar{\xi}}=\int_{\mathbb{R}^{3} \backslash \mathcal{S}}\left[\boldsymbol{\sigma}^{\prime}: \nabla \mathcal{V}\right]: \mathcal{C}:[\boldsymbol{\sigma}: \boldsymbol{\nabla} \mathcal{V}] \mathrm{d} V_{\bar{\xi}}=\boldsymbol{\sigma}: \mathcal{A}^{\sigma}: \boldsymbol{\sigma}^{\prime}
$$

\section{Appendix A.2 Proof of Proposition 3}

Consider first an elastic body $B$ bounded by a smooth closed surface $\partial B=\Gamma$, and let the open surface $S \subset B$ define an interior traction-free crack embedded in $B$. Letting $\boldsymbol{u}^{1}, \boldsymbol{u}^{2}$ denote 
displacements associated with two elastostatic states for the cracked solid $B_{S}:=B \backslash \bar{S}$ (with vanishing body forces assumed for both states), the Maxwell-Betti reciprocity theorem yields

$$
\int_{\Gamma \cup S^{+} \cup S^{-}}\left(\boldsymbol{t}\left[\boldsymbol{u}^{2}\right] \cdot \boldsymbol{u}^{1}-\boldsymbol{t}\left[\boldsymbol{u}^{1}\right] \cdot \boldsymbol{u}^{2}\right) \mathrm{d} S=0
$$

where $S^{ \pm}$denote the two crack faces. Now, let $\boldsymbol{\sigma} \in \mathbb{R}_{\text {sym }}^{3,3}$ denote a constant stress tensor and consider the following particular choices for $\boldsymbol{u}^{1}, \boldsymbol{u}^{2}: \boldsymbol{u}^{1}$ is the (spatially linear) displacement in the uncracked body $B$ created by tractions $\overline{\boldsymbol{t}}:=\boldsymbol{\sigma} \cdot \boldsymbol{n}$ applied on $\Gamma$, while $\boldsymbol{u}^{2}$ is induced by the same applied traction $\overline{\boldsymbol{t}}$ on $\Gamma$ in the cracked body $B_{S}\left(\boldsymbol{u}^{1}\right.$ is thus continuous across $S$ whereas $\boldsymbol{u}^{2}$ is not). Substituting these choices into (A.2) and using the conventions already adopted in e.g. (10) for the normal and displacement discontinuity across $S$, one arrives at

$$
\int_{\Gamma} \overline{\boldsymbol{t}} \cdot\left(\boldsymbol{u}^{1}-\boldsymbol{u}^{2}\right) \mathrm{d} S=-\int_{S}(\boldsymbol{\sigma} \cdot \boldsymbol{n}) \cdot \Delta \boldsymbol{v} \mathrm{d} S
$$

where $\boldsymbol{v}:=\boldsymbol{u}^{2}-\boldsymbol{u}^{1}$ denotes the displacement perturbation induced to $\boldsymbol{u}^{1}$ by the crack, and having used the continuity of $\boldsymbol{u}^{1}$ across $S$.

Letting $E(\boldsymbol{u})$ denote the elastic potential energy of a given state $\boldsymbol{u}$, the integral in the left-hand side of (A.3) is found (by Clapeyron's formula) to be equal to $2 \Delta E$, with $\Delta E:=$ $E\left(\boldsymbol{u}^{2}\right)-E\left(\boldsymbol{u}^{1}\right)$. Moreover, $\boldsymbol{v}$ is (by superposition) the displacement created in $B \backslash S$ by applied tractions $\boldsymbol{t}^{ \pm}=-\boldsymbol{\sigma} \boldsymbol{n}^{ \pm}$on $S^{ \pm}$. In the limiting case where $B$ is unbounded (i.e. if $\operatorname{Diam}(B) \rightarrow \infty$ ), one thus has $\boldsymbol{v}=\boldsymbol{\sigma}: \mathcal{V}$. Upon comparing its right-hand side with (22), the equality (A.3) becomes

$$
\boldsymbol{\sigma}: \mathcal{A}^{\sigma}(S): \boldsymbol{\sigma}=\int_{S}(\boldsymbol{\sigma} \cdot \boldsymbol{n}) \cdot \Delta \boldsymbol{v} \mathrm{d} S=-2 \Delta E
$$

The proof of Proposition 3 now consists in establishing an alternative expression for $\Delta E$ in the case where $B$ is unbounded (note that (A.3) implies that $\Delta E$ remains finite in the limit $\operatorname{Diam}(B) \rightarrow \infty)$, while both $E\left(\boldsymbol{u}^{1}\right)$ and $E\left(\boldsymbol{u}^{2}\right)$ become unbounded). For this purpose, consider a family of homothetical crack shapes $S_{\eta}:=(1+\eta) S$, where $\eta$ is a shape parameter $(\eta=0$ thus corresponding to the original crack $S$ ). For unbounded $B$, the displacement $\boldsymbol{v}_{\eta}$ arising when the crack $S_{\eta}$ is loaded with the same tractions $\boldsymbol{t}^{ \pm}=-\boldsymbol{\sigma} \cdot \boldsymbol{n}^{ \pm}$is such that $\boldsymbol{v}_{\eta}=(1+\eta) \boldsymbol{v}$. Applying the second equality in (A.4) with the crack $S_{\eta}$ and mapping the integral back to $S$ via $\boldsymbol{\xi}_{\eta}=(1+\eta) \boldsymbol{\xi}(\boldsymbol{\xi} \in S)$, one obtains $-2 \Delta E(\eta)=(1+\eta)^{3} \boldsymbol{\sigma}: \mathcal{A}^{\sigma}(S): \boldsymbol{\sigma}$, which upon differentiating w.r.t. $\eta$ and setting $\eta=0$ in the result, yields

$$
\boldsymbol{\sigma}: \mathcal{A}^{\sigma}(S): \boldsymbol{\sigma}=-\left.\frac{2}{3} \frac{\mathrm{d}}{\mathrm{d} \eta} \Delta E(\eta)\right|_{\eta=0}
$$

Now the remaining task is to evaluate the potential energy derivative appearing in (A.5). This exploits a well-known result from linear fracture mechanics, namely the link between this derivative and the energy release rate $G(s)$ at all points of the crack front (parameterized by the arc length $s$ ). Noting that the chosen crack shape transformation $S \mapsto S_{\eta}$ is such that $\mathrm{d} \boldsymbol{\xi}_{\eta} /\left.\mathrm{d} \eta\right|_{\eta=0}=\boldsymbol{\xi}$ for any point $\boldsymbol{\xi} \in S$, this link has here the specific form

$$
\left.\frac{\mathrm{d}}{\mathrm{d} \eta} \Delta E(\eta)\right|_{\eta=0}=-\int_{\partial S} G(s)\left(\left.\boldsymbol{\nu}(s) \cdot \frac{\mathrm{d} \boldsymbol{\xi}_{\eta}(s)}{\mathrm{d} \eta}\right|_{\eta=0}\right) \mathrm{d} s=-\int_{\partial S} G(s)(\boldsymbol{\nu}(s) \cdot \boldsymbol{\xi}(s)) \mathrm{d} s
$$

wherein the energy release rate $G(s)$ corresponds to state $\boldsymbol{u}^{2}$ (or, equivalently, to state $\boldsymbol{v}=\boldsymbol{\sigma}: \mathcal{V}$ ) and $\boldsymbol{\nu}(s)$ is the outward unit normal to $\partial \mathcal{S}$ lying in the tangent plane of $S$. Combining (A.5) and (A.6) yields the desired result (27). 


\section{Appendix B TRANSIENT FUNDAMENTAL SOLUTIONS AND PROOF OF LEMMAS 1,2}

The time convolutions featured in integral equation (12) can be expressed as

$$
\begin{aligned}
& \boldsymbol{U}(\boldsymbol{x}, t, \boldsymbol{\xi}) \star \Delta \ddot{\boldsymbol{v}}_{a, \boldsymbol{z}}(\boldsymbol{\xi}, t)=U_{k i}\left[\boldsymbol{x}, t, \boldsymbol{\xi} \mid\left[\Delta \ddot{\boldsymbol{v}}_{a, \boldsymbol{z}}(\boldsymbol{\xi}, \cdot)\right]_{i}\right] \boldsymbol{e}_{k} \\
& \boldsymbol{\Sigma}(\boldsymbol{x}, t, \boldsymbol{\xi}) \underset{\underline{ }}{=} \mathcal{D}\left[\Delta \boldsymbol{v}_{a, \boldsymbol{z}}\right](\boldsymbol{\xi}, t)=\Sigma_{k i j}\left[\boldsymbol{x}, t, \boldsymbol{\xi} \mid \mathcal{D}\left[\Delta \boldsymbol{v}_{a, \boldsymbol{z}}\right]_{i j \ell}(\boldsymbol{\xi}, \cdot)\right] \boldsymbol{e}_{k} \otimes \boldsymbol{e}_{\ell}
\end{aligned}
$$

where $\boldsymbol{U}[\boldsymbol{x}, t, \boldsymbol{\xi} \mid f]$ and $\boldsymbol{\Sigma}[\boldsymbol{x}, t, \boldsymbol{\xi} \mid f]$ are the time-modulated elastodynamic Green's tensors, defined such that $U_{k i} \boldsymbol{e}_{i}$ and $\Sigma_{k i j} \boldsymbol{e}_{i} \otimes \boldsymbol{e}_{j}$ are the displacement vector and stress tensor at $\boldsymbol{\xi} \in \Omega$ resulting from a point force applied at $\boldsymbol{x}$ in the $k$-direction with prescribed time-varying magnitude $f(t)$ and satisfying boundary conditions (11). Homogeneous initial conditions at $t=0$ and vanishing time modulation $f(t)$ for $t<0$ are assumed, so that $\boldsymbol{U}[\boldsymbol{x}, t, \boldsymbol{\xi} \mid f]$ and $\boldsymbol{\Sigma}[\boldsymbol{x}, t, \boldsymbol{\xi} \mid f]$ have quiescent past. Similarly, let $\boldsymbol{U}_{\infty}[\boldsymbol{x}, t, \boldsymbol{\xi} \mid f]$ and $\boldsymbol{\Sigma}_{\infty}[\boldsymbol{x}, t, \boldsymbol{\xi} ; \boldsymbol{n} \mid f]$ denote the time-modulated full-space fundamental solution, which are given by [30]

$$
\begin{aligned}
\boldsymbol{U}_{\infty}[\boldsymbol{x}, t, \boldsymbol{\xi} \mid f] & =\frac{1}{4 \pi \mu r}[A[\boldsymbol{x}, t, \boldsymbol{\xi} \mid f] \boldsymbol{I}+B[\boldsymbol{x}, t, \boldsymbol{\xi} \mid f](\hat{\boldsymbol{r}} \otimes \hat{\boldsymbol{r}})] \\
\boldsymbol{\Sigma}_{\infty}[\boldsymbol{x}, t, \boldsymbol{\xi} \mid f] & =\frac{1}{4 \pi r^{2}}\left[C[\boldsymbol{x}, t, \boldsymbol{\xi} \mid f](\hat{\boldsymbol{r}} \otimes \boldsymbol{I})+2 D[\boldsymbol{x}, t, \boldsymbol{\xi} \mid f]\left(\hat{\boldsymbol{r}} \cdot \mathcal{I}_{\mathrm{sym}}\right)+2 E[\boldsymbol{x}, t, \boldsymbol{\xi} \mid f](\hat{\boldsymbol{r}} \otimes \hat{\boldsymbol{r}} \otimes \hat{\boldsymbol{r}})\right]
\end{aligned}
$$

where $\boldsymbol{r}=(\boldsymbol{\xi}-\boldsymbol{x}), r=\|\boldsymbol{r}\|, \hat{\boldsymbol{r}}=\boldsymbol{r} / r, \kappa$ is the ratio of bulk wave velocities as defined by

$$
\kappa^{2}=\frac{c_{T}^{2}}{c_{L}^{2}}=\frac{1-2 \nu}{2(1-\nu)}=\frac{\mu}{\lambda+2 \mu}
$$

and with $A=A[\boldsymbol{x}, t, \boldsymbol{\xi} \mid f], \ldots$ defined by

$$
\begin{aligned}
& A[\boldsymbol{x}, t, \boldsymbol{\xi} \mid f]=f\left(t-\frac{r}{c_{T}}\right)+\int_{1}^{\kappa} \eta f\left(t-\frac{\eta r}{c_{T}}\right) \mathrm{d} \eta \\
& B[\boldsymbol{x}, t, \boldsymbol{\xi} \mid f]=-3 A[\boldsymbol{x}, t, \boldsymbol{\xi} \mid f]+2 f\left(t-\frac{r}{c_{T}}\right)+\kappa^{2} f\left(t-\frac{r}{c_{L}}\right) \\
& C[\boldsymbol{x}, t, \boldsymbol{\xi} \mid f]=2 B[\boldsymbol{x}, t, \boldsymbol{\xi} \mid f]-\left(1-2 \kappa^{2}\right)\left\{f\left(t-\frac{r}{c_{L}}\right)+\frac{r}{c_{L}} \dot{f}\left(t-\frac{r}{c_{L}}\right)\right\} \\
& D[\boldsymbol{x}, t, \boldsymbol{\xi} \mid f]=2 B[\boldsymbol{x}, t, \boldsymbol{\xi} \mid f]-f\left(t-\frac{r}{c_{T}}\right)-\frac{r}{c_{T}} \dot{f}\left(t-\frac{r}{c_{T}}\right) \\
& E[\boldsymbol{x}, t, \boldsymbol{\xi} \mid f]=-3 B[\boldsymbol{x}, t, \boldsymbol{\xi} \mid f]-D[\boldsymbol{x}, t, \boldsymbol{\xi} \mid f]-\kappa^{2}\left\{f\left(t-\frac{r}{c_{L}}\right)+\frac{r}{c_{L}} \dot{f}\left(t-\frac{r}{c_{L}}\right)\right\} .
\end{aligned}
$$

Next, define the time-modulated complementary elastodynamic Green's tensor $\boldsymbol{U}_{\mathrm{C}}$ by

$$
\boldsymbol{U}[\boldsymbol{x}, t, \boldsymbol{\xi} \mid f]=\boldsymbol{U}_{\infty}[\boldsymbol{x}, t, \boldsymbol{\xi} \mid f]+\boldsymbol{U}_{\mathrm{C}}[\boldsymbol{x}, t, \boldsymbol{\xi} \mid f]
$$

By virtue of superposition arguments, $\boldsymbol{U}_{\mathrm{C}}$ is governed by an IBVP with vanishing body forces and initial conditions, and (when $\boldsymbol{x} \notin \Gamma$ ) smooth boundary data involving boundary traces $\boldsymbol{U}_{\infty}[\boldsymbol{x}, t, \boldsymbol{\xi} \mid f]\left(\overline{\boldsymbol{\xi}} \in \Gamma_{\mathrm{D}}\right)$ and $\boldsymbol{\Sigma}_{\infty}[\boldsymbol{x}, t, \boldsymbol{\xi} \mid f] \cdot \boldsymbol{n}(\overline{\boldsymbol{\xi}})\left(\overline{\boldsymbol{\xi}} \in \Gamma_{\mathrm{N}}\right)$. Thus, $\boldsymbol{U}_{\mathrm{C}}[\boldsymbol{x}, t, \boldsymbol{\xi} \mid f]$ is bounded in the limit $\boldsymbol{\xi} \rightarrow \boldsymbol{x}$, i.e. the singular behavior of $\boldsymbol{U}[\boldsymbol{x}, t, \boldsymbol{\xi} \mid f]$ at $\boldsymbol{\xi}=\boldsymbol{x}$ is identical to that of its full-space counterpart $\boldsymbol{U}_{\infty}[\boldsymbol{x}, t, \boldsymbol{\xi} \mid f]$, and one has

$$
\boldsymbol{U}_{\mathrm{C}}[\boldsymbol{z}+a \overline{\boldsymbol{x}}, t, \boldsymbol{z}+a \overline{\boldsymbol{\xi}} \mid f]=O(1), \quad \boldsymbol{\Sigma}_{\mathrm{C}}[\boldsymbol{z}+a \overline{\boldsymbol{x}}, t, \boldsymbol{z}+a \overline{\boldsymbol{\xi}} \mid f]=O(1) \quad(a \rightarrow 0)
$$


Proof of Lemma 1. The proof exploits decomposition (B.5). First, upon introducing scaled coordinates (13a) into expression (B.2a) of $\boldsymbol{U}_{\infty}$ and definitions (B.4) of $A[\boldsymbol{x}, t, \boldsymbol{\xi} \mid f]$ and $B[\boldsymbol{x}, t, \boldsymbol{\xi} \mid f]$ (wherein $f(t)=\Delta \ddot{\boldsymbol{v}}_{a, \boldsymbol{z}}(\boldsymbol{\xi}, t)$ according to (B.1a)), it is a simple matter to show that

$$
\boldsymbol{U}_{\infty}(\boldsymbol{x}, t, \boldsymbol{\xi}) \star \Delta \ddot{\boldsymbol{v}}_{a, \boldsymbol{z}}(\boldsymbol{\xi}, t)=\frac{1}{a} \boldsymbol{U}_{\infty, a}(\overline{\boldsymbol{x}}, t, \overline{\boldsymbol{\xi}}) \star \Delta \ddot{\overline{\boldsymbol{v}}}_{a, \boldsymbol{z}}(\overline{\boldsymbol{\xi}}, t)
$$

where $\boldsymbol{U}_{\infty, a}$ is defined by (B.2a) and (B.4) with wave velocities $c_{\mathrm{L}}, c_{\mathrm{T}}$ replaced by rescaled values $c_{\mathrm{L}} / a$ and $c_{\mathrm{T}} / a$. Equation (B.7) and scaling (13b) then imply

$$
\int_{S_{a, \boldsymbol{z}}} \boldsymbol{U}_{\infty}(\boldsymbol{x}, t, \boldsymbol{\xi}) \star \Delta \ddot{\boldsymbol{v}}_{a, \boldsymbol{z}}(\boldsymbol{\xi}, t) \mathrm{d} S_{\xi}=a \int_{\mathcal{S}} \boldsymbol{U}_{\infty, a}(\overline{\boldsymbol{x}}, t, \overline{\boldsymbol{\xi}}) \star \Delta \ddot{\boldsymbol{v}}_{a, \boldsymbol{z}}(\overline{\boldsymbol{\xi}}, t) \mathrm{d} S_{\bar{\xi}}
$$

Moreover, owing to the boundedness (B.6) of the complementary Green's tensor $\boldsymbol{U}_{\mathrm{C}}$, one has, upon using again coordinate scaling (13b):

$$
\int_{S_{a, \boldsymbol{z}}} \boldsymbol{U}_{\mathrm{C}}(\boldsymbol{x}, t, \boldsymbol{\xi}) \star \Delta \ddot{\boldsymbol{v}}_{a, \boldsymbol{z}}(\boldsymbol{\xi}, t) \mathrm{d} S_{\xi}=O\left(a^{2}\right)\left\|\Delta \ddot{\overline{\boldsymbol{v}}}_{a, \boldsymbol{z}}(\cdot, t)\right\|_{L^{1}(\mathcal{S})}
$$

The desired identity (15b) then follows from combining (B.8) and (B.9). Identity (15a) is established in a similar way, noting that

$$
\boldsymbol{\Sigma}_{\infty}(\boldsymbol{x}, t, \boldsymbol{\xi}) \triangleq \mathcal{D} \Delta \boldsymbol{v}_{a, \boldsymbol{z}}(\boldsymbol{\xi}, t)=\frac{1}{a^{3}} \boldsymbol{\Sigma}_{\infty, a}(\overline{\boldsymbol{x}}, t, \overline{\boldsymbol{\xi}}) \triangleq \mathcal{D} \Delta \overline{\boldsymbol{v}}_{a, \boldsymbol{z}}(\overline{\boldsymbol{\xi}}, t)
$$

with the $a^{-3}$ behavior resulting from the combined effect of the $\|\boldsymbol{\xi}-\boldsymbol{x}\|^{-2}$ singularity of $\boldsymbol{\Sigma}_{\infty}$ and the following behavior of the operator $\mathcal{D}$ under the scaling (13a):

$$
\mathcal{D} \boldsymbol{w}(\boldsymbol{\xi}, t)=\frac{1}{a} \mathcal{D} \overline{\boldsymbol{w}}(\overline{\boldsymbol{\xi}}, t)
$$

Proof of Lemma 2. Since the proposed ansatz (16) is, by assumption, differentiable w.r.t. $t$, it is appropriate to investigate the behavior of $\boldsymbol{U}_{\infty}$ defined by (B.2a) and (B.4) for a differentiable time modulation $f$. Introducing the decomposition $f(\tau)=f(t)+(f(\tau)-f(t))=$ $f(t)+\Delta f(\tau)$, one has

$$
\begin{aligned}
& C[\overline{\boldsymbol{x}}, t, \overline{\boldsymbol{\xi}} \mid f]=\kappa^{2} f(t)+C[\overline{\boldsymbol{x}}, t, \overline{\boldsymbol{\xi}} \mid \Delta f] \\
& D[\overline{\boldsymbol{x}}, t, \overline{\boldsymbol{\xi}} \mid f]=-\kappa^{2} f(t)+D[\overline{\boldsymbol{x}}, t, \overline{\boldsymbol{\xi}} \mid \Delta f] \\
& E[\overline{\boldsymbol{x}}, t, \overline{\boldsymbol{\xi}} \mid f]=-\frac{3}{2}\left(1-\kappa^{2}\right) f(t)+E[\overline{\boldsymbol{x}}, t, \overline{\boldsymbol{\xi}} \mid \Delta f]
\end{aligned}
$$

Substituting the above values into (B.2b) yields the decomposition

$$
\boldsymbol{\Sigma}_{\infty}[\overline{\boldsymbol{x}}, t, \overline{\boldsymbol{\xi}} \mid f]=\boldsymbol{\Sigma}_{\infty}(\overline{\boldsymbol{\xi}}-\overline{\boldsymbol{x}}) f(t)+\boldsymbol{\Sigma}_{\infty}[\overline{\boldsymbol{x}}, t, \overline{\boldsymbol{\xi}} \mid \Delta f]
$$

where $\boldsymbol{\Sigma}_{\infty}(\overline{\boldsymbol{r}})$, given by

$$
\Sigma_{\infty}(\overline{\boldsymbol{r}})=\frac{1}{4 \pi \bar{r}^{2}}\left[\kappa^{2}\left(\hat{\boldsymbol{r}} \otimes \boldsymbol{I}-2 \hat{\overline{\boldsymbol{r}}} \cdot \mathcal{I}_{\mathrm{sym}}\right)+3\left(\kappa^{2}-1\right) \hat{\overline{\boldsymbol{r}}} \otimes \hat{\boldsymbol{r}} \otimes \hat{\boldsymbol{r}}\right]
$$

is in fact the elastostatic Kelvin fundamental stress. Decomposition (B.11) in particular holds for $\Sigma_{\infty, a}[\ldots \mid f]$ defined by replacing velocities $c_{\mathrm{L}}, c_{\mathrm{T}}$ with the rescaled values $c_{\mathrm{L}} / a, c_{\mathrm{T}} / a$ in 
$\Sigma_{\infty}[\ldots \mid f]$. Owing to the assumed differentiability of $f$, one easily shows that

$$
\left\|\boldsymbol{\Sigma}_{\infty, a}[\overline{\boldsymbol{x}}, t, \overline{\boldsymbol{\xi}} \mid \Delta f]\right\|=O(a) \quad(a \rightarrow 0)
$$

Since $\boldsymbol{\Sigma}_{\infty}(\overline{\boldsymbol{r}}) f(t)$ is unaffected by the wave velocity rescaling, decomposition (B.11) implies

$$
\boldsymbol{\Sigma}_{\infty, a}[\overline{\boldsymbol{x}}, t, \overline{\boldsymbol{\xi}} \mid f]=\boldsymbol{\Sigma}_{\infty}(\overline{\boldsymbol{x}}, \overline{\boldsymbol{\xi}}) f(t)+o(1) \quad(a \rightarrow 0)
$$

Consequently, noting that combining ansatz (16) with (B.10) implies

$$
\mathcal{D} \Delta \overline{\boldsymbol{v}}_{a, \boldsymbol{z}}(\overline{\boldsymbol{\xi}}, t)=\boldsymbol{\sigma}[\boldsymbol{u}](\boldsymbol{z}, t): \mathcal{D} \Delta \mathcal{V}(\overline{\boldsymbol{\xi}})+o(1), \quad(a \rightarrow 0)
$$

equating $f(t)$ to the components of the above expansion of $\mathcal{D} \Delta \overline{\boldsymbol{v}}_{a, \boldsymbol{z}}(\overline{\boldsymbol{\xi}}, t)$ according to (B.1b), and using (B.13) one finds that

$$
f_{\mathcal{S}} \boldsymbol{\Sigma}_{\infty, a}(\overline{\boldsymbol{x}}, t, \overline{\boldsymbol{\xi}}) \underset{\star}{=} \mathcal{D} \Delta \overline{\boldsymbol{v}}_{a, \boldsymbol{z}}(\overline{\boldsymbol{\xi}}, t) \mathrm{d} S_{\bar{\xi}}=\sigma_{i j}[\boldsymbol{u}](\boldsymbol{z}, t) \int_{\mathcal{S}} \boldsymbol{\Sigma}_{\infty}(\overline{\boldsymbol{\xi}}-\overline{\boldsymbol{x}}): \mathcal{D}\left[\Delta \boldsymbol{V}^{i j}\right](\overline{\boldsymbol{\xi}}) \mathrm{d} S_{\bar{\xi}}+o(1)
$$

The desired expansion (17a) finally follows from combining the above estimate with identity (15b).

Finally, the estimate (17b) stems directly from plugging ansatz (16) into (15b) and the assumed twice-differentiability of $f$.

\section{References}

[1] Abramowitz, M., Stegun, I. Handbook of mathematical functions. Dover Publications (1972).

[2] Achenbach, J. D. Reciprocity in elastodynamics. Cambridge University Press (2003).

[3] Ammari, H., Guadarrama Bustos, L., Garapon, P., Kang, H. Transient anomaly imaging by the acoustic radiation force. J. Diff. Eq., 249:1579-1595 (2010).

[4] Ammari, H., Kang, H. Reconstruction of small inhomogeneities from boundary measurements. Springer-Verlag (2004).

[5] Ammari, H., Kang, H. Reconstruction of elastic inclusions of small volume via dynamic measurements. Appl. Math. Opt., 54:223-235 (2006).

[6] Ammari, H., Kang, H. Polarization and moment tensors with applications to inverse problems and effective medium theory, vol. 162. Springer-Verlag (2007).

[7] Ammari, H., Kang, H., Lee, H., Park, W. K. Asymptotic Imaging of perfectly conducting cracks. SIAM J. Sci. Comput., 32:894-922 (2010).

[8] Ammari, H., Kang, H., Nakamura, G., Tanuma, K. Complete asymptotic expansions of solutions of the system of elastostatics in the presence of an inclusion of small diameter and detection of an inclusion. J. Elast., 67:97-129 (2002).

[9] Amstutz, S., Dominguez, N. Topological sensitivity analysis in the context of ultrasonic nondestructive testing. Engineering Analysis with Boundary Elements, 32:936-947 (2008).

[10] Amstutz, S., Horchani, I., Masmoudi, M. Crack detection by the topological gradient method. Control and Cybernetics, 34:81-101 (2005).

[11] Amstutz, S., Takahashi, T., Vexler, B. Topological sensitivity analysis for time-dependent problems. ESAIM: Control, Optimisation and Calculus of Variations, 14:227-255 (2008).

[12] Andrieux, S., Ben Abda, A., Bui, H. D. Reciprocity principle and crack detection. Inverse Problems, 15:59-66 (1999). 
[13] Bellis, C., Bonnet, M. A FEM-based topological sensitivity approach for fast qualitative identification of buried cavities from elastodynamic overdetermined boundary data. Int. J. Solids Struct., 47:1221-1242 (2010).

[14] Ben Abda, A., Ben Ameur, H., Jaoua, M. Identification of 2D cracks by elastic boundary measurements. Inverse Problems, 15:67-77 (1999).

[15] Bonnet, M. Boundary integral equations methods for solids and fluids. Wiley (1999).

[16] Bonnet, M. Topological sensitivity for 3D elastodynamics and acoustic inverse scattering in the time domain. Comput. Methods Appl. Mech. Engrg., 195:5239-5254 (2006).

[17] Bonnet, M., Constantinescu, A. Inverse problems in elasticity. Inverse Problems, 21:R1-R50 (2005).

[18] Bonnet, M., Delgado, G. The topological derivative in anisotropic elasticity (2012, under review).

[19] Bonnet, M., Guzina, B. B. Sounding of finite solid bodies by way of topological derivative. Int. J. Num. Meth. in Eng., 61:2344-2373 (2004).

[20] Bui, H. D. Fracture mechanics: Inverse problems and solutions. Springer (2006).

[21] Bui, H. D, Constantinescu, A., Maigre, H. Numerical identification of linear cracks in 2D elastodynamics using instantaneous reciprocity gap. Inverse Problems, 20:993-1001 (2004).

[22] Cakoni, F., Colton, D. The linear sampling method for cracks. Inverse Problems, 19:279-295 (2003).

[23] Cakoni, F., Colton, D. Qualitative methods in inverse scattering theory. Springer-Verlag (2006).

[24] Cast3M: A general purpose code for solving partial differential equations by the finite element method, developed by the French Atomic Energy Commission. http://www-cast3m.cea.fr/ (2003).

[25] Cedio-Fengya, D. J., Moskow, S., Vogelius, M. Identification of conductivity imperfections of small diameter by boundary measurements. Continuous dependence and computational reconstruction. Inverse Problems, 14:553-595 (1998).

[26] Chen, Y. Z., Hasebe, N., Lee, K. Y. Multiple crack problems in elasticity. WIT Press (2003).

[27] Colton, D., Kirsch, A. A simple method for solving inverse scattering problems in the resonance region. Inverse Problems, 12:383-393 (1996).

[28] Colton, D., Kress, R. Inverse acoustic and electromagnetic scattering theory. Springer Verlag (1998).

[29] Dominguez, N., Gibiat, V., Esquerré, Y. Time domain topological gradient and time reversal analogy: an inverse method for ultrasonic target detection. Wave Motion, 42:31-52 (2005).

[30] Eringen, A. C., Suhubi, E. S. Elastodynamics (vol II - linear theory). Academic Press (1975).

[31] Eschenauer, H. A., Kobelev, V. V., Schumacher, A. Bubble method for topology and shape optimization of structures. Structural Optimization, 8:42-51 (1994).

[32] Gallego, R., Rus, G. Identification of cracks and cavities using the topological sensitivity boundary integral equation. Comp. Mech., 33:154-163 (2004).

[33] Garreau, S., Guillaume, P., Masmoudi, M. The topological asymptotic for PDE systems: the elasticity case. SIAM J. Control Optim., 39:1756-1778 (2001).

[34] Gradshteyn, I. S., Ryzhik, I. M. Tables of integrals, series and products (seventh edition). Elsevier (2007). 
[35] Guzina, B. B., Bonnet, M. Small-inclusion asymptotic of misfit functionals for inverse problems in acoustics. Inverse Problems, 22:1761-1785 (2006).

[36] Guzina, B. B., Chikichev, I. From imaging to material identification: a generalized concept of topological sensitivity. J. Mech. Phys. Solids, 55:245-279 (2007).

[37] Haug, E. J., Choi, K. K., Komkov, V. Design Sensitivity Analysis of Structural Systems. Academic Press (1986).

[38] Hughes, T. J. R. The finite element method - linear static and dynamic finite element analysis. Prentice Hall, Englewood Cliffs, New Jersey, USA (1987).

[39] Ikehata, M. Inverse crack problem and probe method. Cubo, 8:29-40 (2006).

[40] Jackowska-Strumillo, L., Sokolowski, J., Zochowski, A. Topological optimization and inverse problems. Comput. Assisted Mech. Eng. Sci., 10(2):163-176 (2002).

[41] Kassir, M., Sih, G. C. Three-dimensional crack problems: a new selection of cracks solutions in three-dimensional elasticity. Noordhoof International Pub. (1975).

[42] Kassir, M. K., Sih, G. C. Three-dimensional stress distribution around an elliptical crack under arbitrary loadings. ASME J. Appl. Mech., 33:601-611 (1966).

[43] Kokotov, A., Plamenevskii, B. On the asymptotics of solutions to the Neumann problem for hyperbolic systems in domains with conical points. St. Petersburg Math. J., 16:477-506 (2005).

[44] Maz'ya, V., Nazarov, S. A., Plamenevskii, B. A. Asymptotic theory of elliptic boundary value problems under a singular perturbation of the domains (vols. 1 and 2). Birkhaüser (2000).

[45] Mehrabadi, M. M., Cowin, S. C. Eigentensors of linear anisotropic elastic materials. Quart. J. Mech. Appl. Math., 43:15-41 (1990).

[46] Nintcheu Fata, S., Guzina, B. B. A linear sampling method for near-field inverse problems in elastodynamics. Inverse Problems, 20:713-736 (2004).

[47] Potthast, R. A survey on sampling and probe methods for inverse problems. Inverse Problems, 22:R1-R47 (2006).

[48] Rovati, M., Taliercio, A. Stationarity of the strain energy density for some classes of anisotropic solids. Int. J. Solids Struct., 40:3043-6075 (2003).

[49] Rus, G., Wooh, S. C., Gallego, R. Processing of ultrasonic array signals for characterizing defects. Part II: experimental work. IEEE Trans. Ultrason., Ferroelec., Freq. Contr., 54:2139-2145 (2007).

[50] Sevostianov, I. Thermal conductivity of a material containing cracks of arbitrary shape. Int. J. Eng. Sci., 44:513-528 (2006).

[51] Sokolowski, J., Zochowski, A. On the topological derivative in shape optimization. SIAM J. Control Optim., 37:1251-1272 (1999).

[52] Stavroulakis, G. E. Inverse and crack identification problems in engineering mechanics. Springer (2001).

[53] Tada, H., Paris, P. C., Irwin, G. R. The stress analysis of cracks handbook. Professional Engineering Publishing (2000).

[54] Wang, X. D., Huang, G. L. Identification of embedded cracks using back-propagating elastic waves. Inverse Problems, 20:1393-1409 (2004).

[55] Wheeler, L. T., Sternberg, E. Some theorems in classical elastodynamics. Arch. Rat. Mech. Anal., 31:51-90 (1968). 